\title{
COUPLING DARCY AND STOKES EQUATIONS FOR POROUS MEDIA WITH CRACKS
}

\author{
Christine Bernardi ${ }^{1},{\text { Frédéric } \text { Hecht }^{1} \text { and Olivier Pironneau }}^{1}$
}

\begin{abstract}
In order to handle the flow of a viscous incompressible fluid in a porous medium with cracks, the thickness of which cannot be neglected, we consider a model which couples the Darcy equations in the medium with the Stokes equations in the cracks by a new boundary condition at the interface, namely the continuity of the pressure. We prove that this model admits a unique solution and propose a mixed formulation of it. Relying on this formulation, we describe a finite element discretization and derive a priori and a posteriori error estimates. We present some numerical experiments that are in good agreement with the analysis.
\end{abstract}

Mathematics Subject Classification. 65N30, 65N50, 76D07, 76S05.

Received: December 2, 2002. Revised: March 2, 2003.

\section{INTRODUCTION}

The modelling of cracks is important for the numerical simulation of geophysical systems. The difficulty lies in the fact that there is a large difference of scales between the porous media proper and the crack where the flow is much faster. In the $d$-dimensional porous medium $(d=2$ or 3$)$ Darcy equations are used and the velocity of the flow is the gradient of the hydrostatic potential. Most often the thickness of the cracks is small enough to be neglected and each crack is only modelled by a manifold of dimension $d-1$ which is taken out of the domain occupied by the porous medium. However, for thicker cracks, a new model must be used: provided the effect of the sand filling the crack is neglected, the flow is Newtonian and at creeping speed it satisfies the Stokes equations. The problem of the numerical matching of both Darcy and Stokes equations is the subject of this study. There are other important applications beside cracks such as the seepage of water in sand, either from a lake into the ground or from the sea in the sand beach [21]; but there the flow in the liquid part satisfies the Navier-Stokes equations rather than the Stokes ones.

Interface conditions are a matter of controversy. From the mathematical standpoint homogenization reveals a boundary layer and the interface conditions are far field approximations of this boundary layer. From a physical point of view, conservation of fluid imposes continuity of the normal velocities at the interface. Similarly, conservation of momentum enforces conservation of the normal stress. Such interface conditions are studied for instance in $[11,23]$ and ([9], Sect. 4.5), with a further equation on the tangential stress: its jump is assumed to be proportional to the slip velocity. But this requires a modelling constant which must be measured.

Keywords and phrases. Darcy and Stokes equations, finite elements, error estimates.

1 Laboratoire Jacques-Louis Lions, CNRS \& Université Pierre et Marie Curie, B.C. 187, 4 place Jussieu, 75252 Paris Cedex 05, France. bernardi@ann.jussieu.fr; hecht@ann.jussieu.fr; pironneau@ann.jussieu.fr 
In this paper, we use a different approach, relying on the fact that on the Darcy flow side the stress is normal and proportional to the pressure while the normal stress is $\left(\mu\left(\nabla \boldsymbol{u}+\nabla \boldsymbol{u}^{T}\right)+p \operatorname{Id}\right) \cdot \boldsymbol{n}$ in the Stokes or Navier-Stokes side. At high Reynolds number, $\mu$ is small and the normal stress is dominated by the pressure because $\nabla \boldsymbol{u}$ remains bounded (this is not proved but can be observed). Therefore a boundary condition which imposes continuity of the pressure is an approximation at high Reynolds number of the continuity of the normal stress. By deciding to work with this condition we avoid the modelling of the third interface condition. Indeed it comes directly from the necessity that the rotational of the velocity is zero on the interface, otherwise the continuity of the pressure cannot be enforced. So while the condition chosen in this paper is wrong from the physical point of view, it is an approximation of the real - yet hypothetical - physical condition which does not require any further modelling. In principle it should be used with the Navier-Stokes equation and not with the Stokes equation on the viscous flow side. Here we present the analysis for the Stokes problem, mostly for the sake of clarity, since the extension to the nonlinear Navier-Stokes equations is technical only.

It is not difficult to establish existence and uniqueness for our model in the continuous case; the main difficulties are linked to the discretization. In [21] a fictitious domain approach is chosen and the interface conditions are treated by a penalty-like method. In [22], a conforming finite element method is used and convergence is established by choosing discrete spaces which satisfy the inf-sup condition, like for the Stokes problem, but within this new framework. In [12,13], a domain decomposition is introduced, with one subdomain for the medium and another one for the fluid, and an iterative procedure for solving the resulting system is analyzed (see also [9], Sect. 4.5.5). Here we have chosen to discretize the system by a mixed finite element method since the associated variational formulation has three advantages:

- it is fully equivalent to the system of partial differential equations, i.e. the equivalence does not require any further regularity of the solution;

- it handles all the interface conditions except one;

- it allows to use the same discretization space for the variables in both regions.

A priori and a posteriori estimates are given. They are not optimal with respect to the approximation error but they are almost optimal for the discretization, as shown by a counter-example.

Some preliminary numerical tests are given in the two-dimensional case. They confirm the theory and show that the method is feasible and can be used for real life applications.

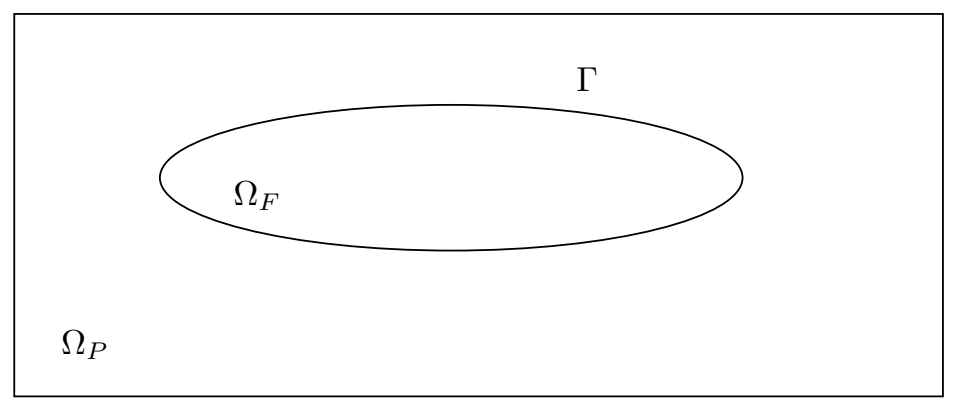

Figure 1. An example of geometry.

An outline of the paper is as follows.

- Section 2 is devoted to the description of the model and to the study of its standard formulation.

- In Section 3, we write the mixed formulation of the model and prove its well-posedness.

- In Section 4, the discrete problem relying on this last formulation is written. Its well-posedness is proven, together with some a priori error estimates.

- In Section 5, we introduce some error indicators and derive a posteriori error estimates.

- Numerical experiments are presented in Section 6.

- An appendix is devoted to the proof of a rather technical result. 


\section{DESCRIPTION AND ANALYSis OF THE MODEL}

Let $\Omega$ and $\Omega_{F}$ be bounded connected open domains in $\mathbb{R}^{d}, d=2$ or 3 , with Lipschitz-continuous boundaries, such that $\bar{\Omega}_{F}$ is contained in $\Omega$. For simplicity, we also assume that $\Omega_{F}$ is simply connected and has a connected boundary. We set: $\Omega_{P}=\Omega \backslash \bar{\Omega}_{F}$ and we denote by $\Gamma=\partial \Omega_{F}$ the interface between $\Omega_{P}$ and $\Omega_{F}$ (this is illustrated in Fig. 1). Let also $\boldsymbol{n}$ stand for the unit outward normal vector to $\Omega_{P}$ on its boundary $\partial \Omega_{P}$.

We consider the following system of equations

$$
\begin{cases}\mu \boldsymbol{u}+\operatorname{grad} p=\boldsymbol{f} & \text { in } \Omega_{P}, \\ -\nu \Delta \boldsymbol{u}+\operatorname{grad} p=\boldsymbol{f} & \text { in } \Omega_{F}, \\ \operatorname{div} \boldsymbol{u}=0 & \text { in } \Omega_{P} \text { and } \Omega_{F}, \\ \boldsymbol{u} \cdot \boldsymbol{n}=0 & \text { on } \partial \Omega, \\ \left(\boldsymbol{u}_{\mid \Omega_{P}}-\boldsymbol{u}_{\mid \Omega_{F}}\right) \cdot \boldsymbol{n}=0 & \text { on } \Gamma, \\ p_{\mid \Omega_{P}}-p_{\mid \Omega_{F}}=0 & \text { on } \Gamma, \\ \operatorname{curl} \boldsymbol{u}_{\mid \Omega_{F}} \times \boldsymbol{n}=\mathbf{0} & \text { on } \Gamma .\end{cases}
$$

Here, the unknowns are the velocity $\boldsymbol{u}$ and the pressure $p$, while $\boldsymbol{f}$ represents a density of body forces. The parameters $\mu$ and $\nu$ are positive constants and denote the ratio of the viscosity of the fluid to the permeability of the medium, respectively the viscosity of the fluid (note that the parameter $\mu^{-1}$ is also called the porosity).

In order to write a variational formulation of problem (2.1), we first introduce the space

$$
H(\operatorname{div}, \Omega)=\left\{\boldsymbol{v} \in L^{2}(\Omega)^{d} ; \operatorname{div} \boldsymbol{v} \in L^{2}(\Omega)\right\},
$$

provided with the norm

$$
\|\boldsymbol{v}\|_{H(\operatorname{div}, \Omega)}=\left(\|\boldsymbol{v}\|_{L^{2}(\Omega)^{d}}^{2}+\|\operatorname{div} \boldsymbol{v}\|_{L^{2}(\Omega)}^{2}\right)^{\frac{1}{2}} .
$$

We recall that $H(\operatorname{div}, \Omega)$ is a Hilbert space and also that the trace operator: $\boldsymbol{v} \mapsto \boldsymbol{v} \cdot \boldsymbol{n}$ is continuous from $H(\operatorname{div}, \Omega)$ onto $H^{-\frac{1}{2}}(\partial \Omega)$. We denote by $H_{0}(\operatorname{div}, \Omega)$ the space of functions $\boldsymbol{v}$ in $H(\operatorname{div}, \Omega)$ such that $\boldsymbol{v} \cdot \boldsymbol{n}$ vanishes on $\partial \Omega$ (and we use analogous spaces with $\Omega$ replaced by $\Omega_{P}$ and $\Omega_{F}$ ). Finally, we introduce the space

$$
H(\operatorname{curl}, \Omega)=\left\{\boldsymbol{v} \in L^{2}(\Omega)^{d} ; \operatorname{curl} \boldsymbol{v} \in L^{2}(\Omega)^{\frac{d(d-1)}{2}}\right\},
$$

also provided with the graph norm of the curl operator.

Note moreover that, for all functions $\boldsymbol{v}$ in $H(\operatorname{div}, \Omega)$, the jump $\left(\boldsymbol{v}_{\mid \Omega_{P}}-\boldsymbol{v}_{\mid \Omega_{F}}\right) \cdot \boldsymbol{n}$ vanishes on $\Gamma$. We now consider the space

$$
X=\left\{\boldsymbol{v} \in H(\operatorname{div}, \Omega) ; \operatorname{curl} \boldsymbol{v}_{\mid \Omega_{F}} \in L^{2}\left(\Omega_{F}\right)^{\frac{d(d-1)}{2}} \text { and } \boldsymbol{v} \cdot \boldsymbol{n}=0 \text { on } \partial \Omega\right\},
$$

provided with the norm

$$
\|\boldsymbol{v}\|_{X}=\left(\|\boldsymbol{v}\|_{H(\operatorname{div}, \Omega)}^{2}+\|\operatorname{curl} \boldsymbol{v}\|_{L^{2}\left(\Omega_{F} \frac{d(d-1)}{2}\right.}^{2}\right)^{\frac{1}{2}} .
$$

It is readily checked that $X$ is a Hilbert space. We also need the space $L_{0}^{2}(\Omega)$ of functions in $L^{2}(\Omega)$ which have a null integral on $\Omega$.

Next, we introduce the bilinear forms

$$
a(\boldsymbol{u}, \boldsymbol{v})=\mu \int_{\Omega_{P}} \boldsymbol{u} \cdot \boldsymbol{v} \mathrm{d} \boldsymbol{x}+\nu \int_{\Omega_{F}} \operatorname{curl} \boldsymbol{u} \cdot \operatorname{curl} \boldsymbol{v} \mathrm{d} \boldsymbol{x}, \quad b(\boldsymbol{v}, q)=-\int_{\Omega}(\operatorname{div} \boldsymbol{v}) p \mathrm{~d} \boldsymbol{x} .
$$


Obviously, these forms are continuous on $X \times X$ and $X \times L_{0}^{2}(\Omega)$, respectively. For any data $f$ in $L^{2}(\Omega)^{d}$, we consider the variational problem

Find $(\boldsymbol{u}, p)$ in $X \times L_{0}^{2}(\Omega)$ such that

$$
\begin{aligned}
& \forall \boldsymbol{v} \in X, \quad a(\boldsymbol{u}, \boldsymbol{v})+b(\boldsymbol{v}, p)=\int_{\Omega} \boldsymbol{f} \cdot \boldsymbol{v} \mathrm{d} \boldsymbol{x}, \\
& \forall q \in L_{0}^{2}(\Omega), \quad b(\boldsymbol{u}, q)=0 .
\end{aligned}
$$

Remark 2.1. Thanks to the formula

$$
-\Delta \boldsymbol{u}=\operatorname{curl}(\operatorname{curl} \boldsymbol{u})-\operatorname{grad}(\operatorname{div} \boldsymbol{u})
$$

when the domain $\Omega_{F}$ is of class $\mathscr{C}^{1,1}$ and when the function $\boldsymbol{f}$ belongs to $L^{2}(\Omega)^{d}$ and is such that its restriction to $\Omega_{F}$ belong to $H\left(\mathbf{c u r l}, \Omega_{F}\right)$, it is readily checked that any solution $(\boldsymbol{u}, p)$ of $(2.5)$ such that $p_{\mid \Omega_{F}}$ belongs to $H^{1}\left(\Omega_{F}\right)$, is a solution of (2.1) (see [4], Thm. 1.9): indeed, the first three lines are satisfied in the distribution sense, the fourth and fifth line are satisfied in $H^{-\frac{1}{2}}(\partial \Omega)$ and $H^{-\frac{1}{2}}(\Gamma)$, respectively; the sixth line is satisfied in $H^{\frac{1}{2}}(\Gamma)$ thanks to the assumption on $p$ since it can be checked from the first line in (2.1) that $p_{\mid \Omega_{P}}$ belongs to $H^{1}\left(\Omega_{P}\right)$; finally, the last line is satisfied in $H^{-\frac{1}{2}}(\Gamma)^{\frac{d(d-1)}{2}}$ since, thanks to the assumption on $\Omega_{F}$ and $\boldsymbol{f}$, the function $\boldsymbol{\omega}=\operatorname{curl} \boldsymbol{u}_{\mid \Omega_{F}}$ is such that $\Delta \boldsymbol{\omega}$ belongs to $L^{2}\left(\Omega_{F}\right)^{\frac{d(d-1)}{2}}$, hence has a trace in $H^{-\frac{1}{2}}(\Gamma)^{\frac{d(d-1)}{2}}$. Conversely, it can be checked by the arguments in ([4], Thm. 1.8), that any solution ( $\boldsymbol{u}, p)$ of problem (2.1) in $\mathscr{C}^{2}(\bar{\Omega}) \times \mathscr{C}^{1}(\bar{\Omega})$ is a solution of problem (2.5). However, the full equivalence of problems $(2.1)$ and $(2.5)$ would require the density of the space $\mathscr{D}(\Omega)^{d}$ in $X$, which seems unknown.

In order to investigate the well-posedness of problem (2.5), we first introduce the kernel

$$
V=\left\{\boldsymbol{v} \in X ; \forall q \in L_{0}^{2}(\Omega), b(\boldsymbol{v}, q)=0\right\} .
$$

Since functions in $X$ have their divergence in $L_{0}^{2}(\Omega)$, this space is equivalently given by

$$
V=\{\boldsymbol{v} \in X ; \operatorname{div} \boldsymbol{v}=0 \text { in } \Omega\} .
$$

Moreover, for any solution $(\boldsymbol{u}, p)$ of problem (2.5), the velocity $\boldsymbol{u}$ belongs to $V$ and satisfies

$$
\forall \boldsymbol{v} \in V, \quad a(\boldsymbol{u}, \boldsymbol{v})=\int_{\Omega} \boldsymbol{f} \cdot \boldsymbol{v} \mathrm{d} \boldsymbol{x} .
$$

So we must prove the ellipticity of the form $a(\cdot, \cdot)$ on $V$.

Let us first note that, if a function $\boldsymbol{v}$ in $V$ satisfies $a(\boldsymbol{v}, \boldsymbol{v})=0$, its restriction to $\Omega_{P}$ vanishes, so that its restriction to $\Omega_{F}$ satisfies

$$
\operatorname{div} \boldsymbol{v}_{\mid \Omega_{F}}=0 \quad \text { in } \Omega_{F}, \quad \operatorname{curl} \boldsymbol{v}_{\mid \Omega_{F}}=0 \quad \text { in } \Omega_{F}, \quad \boldsymbol{v}_{\mid \Omega_{F}} \cdot \boldsymbol{n}=0 \quad \text { on } \Gamma .
$$

Since $\Omega_{F}$ is simply-connected, that yields ([3], Prop. 3.14), that it is zero. So the form $a(\cdot, \cdot)$ is semi-positive definite. However, since the imbedding of the space of functions in $H\left(\operatorname{div} ; \Omega_{F}\right) \cap H\left(\operatorname{curl} ; \Omega_{F}\right)$ into $L^{2}\left(\Omega_{F}\right)^{d}$ is not compact ([3], Prop. 2.7), the ellipticity of $a(\cdot, \cdot)$ cannot be derived from the Peetre-Tartar lemma ([18], Chap. I, Thm. 2.1), and requires a further argument.

Lemma 2.2. There exists a constant $\alpha_{0}>0$ such that

$$
\forall \boldsymbol{v} \in X, \quad\|\boldsymbol{v}\|_{L^{2}\left(\Omega_{F}\right)^{d}} \leq \alpha_{0}\left(\|\boldsymbol{v}\|_{L^{2}\left(\Omega_{P}\right)^{d}}^{2}+\|\operatorname{div} \boldsymbol{v}\|_{L^{2}(\Omega)}^{2}+\|\operatorname{curl} \boldsymbol{v}\|_{L^{2}\left(\Omega_{F}\right)^{\frac{d(d-1)}{2}}}^{2}\right)^{\frac{1}{2}} .
$$


Proof. The proof is only given in dimension $d=3$ since it is more complex in this case. We have

$$
\|\boldsymbol{v}\|_{L^{2}\left(\Omega_{F}\right)^{d}}=\sup _{\boldsymbol{g} \in L^{2}\left(\Omega_{F}\right)^{d}, \boldsymbol{g} \neq \mathbf{0}} \frac{\int_{\Omega_{F}} \boldsymbol{g} \cdot \boldsymbol{v} \mathrm{d} \boldsymbol{x}}{\|\boldsymbol{g}\|_{L^{2}\left(\Omega_{F}\right)^{d}}}
$$

Next, we use the following property ([3], Sect. 3.e): since $\Omega_{F}$ is simply-connected, each function $\boldsymbol{g}$ in $L^{2}\left(\Omega_{F}\right)^{d}$ can be written: $\boldsymbol{g}=\operatorname{grad} \chi+\operatorname{curl} \boldsymbol{\varphi}$, where $\chi$ belongs to $H^{1}\left(\Omega_{F}\right) \cap L_{0}^{2}\left(\Omega_{F}\right)$ and $\boldsymbol{\varphi}$ is divergence-free on $\Omega_{F}$ and has a zero tangential trace on $\partial \Omega_{F}$. Note that this yields the property

$$
\|\boldsymbol{g}\|_{L^{2}\left(\Omega_{F}\right)^{d}}^{2}=\|\operatorname{grad} \chi\|_{L^{2}\left(\Omega_{F}\right)^{d}}^{2}+\|\operatorname{curl} \varphi\|_{L^{2}\left(\Omega_{F}\right)^{d}}^{2}
$$

1) We have

$$
\int_{\Omega_{F}} \operatorname{grad} \chi \cdot \boldsymbol{v} \mathrm{d} \boldsymbol{x}=-\int_{\Omega_{F}}(\operatorname{div} \boldsymbol{v}) \chi \mathrm{d} \boldsymbol{x}+\int_{\Gamma} \boldsymbol{v} \cdot \boldsymbol{n} \chi \mathrm{d} \boldsymbol{\tau} \leq\|\operatorname{div} \boldsymbol{v}\|_{L^{2}\left(\Omega_{F}\right)}\|\chi\|_{L^{2}\left(\Omega_{F}\right)}+\|\boldsymbol{v} \cdot \boldsymbol{n}\|_{H^{-\frac{1}{2}}(\Gamma)}\|\chi\|_{H^{\frac{1}{2}}(\Gamma)} .
$$

We also deduce from the trace theorem on $H\left(\operatorname{div}, \Omega_{P}\right)$ that

$$
\|\boldsymbol{v} \cdot \boldsymbol{n}\|_{H^{-\frac{1}{2}(\Gamma)}} \leq c\left(\|\boldsymbol{v}\|_{L^{2}\left(\Omega_{P}\right)^{d}}^{2}+\|\operatorname{div} \boldsymbol{v}\|_{L^{2}\left(\Omega_{P}\right)}^{2}\right)^{\frac{1}{2}}
$$

By combining all this and using the trace theorem on $H^{1}\left(\Omega_{F}\right)$ together with Bramble-Hilbert inequality, we obtain

$$
\int_{\Omega_{F}} \operatorname{grad} \chi \cdot \boldsymbol{v} \mathrm{d} \boldsymbol{x} \leq c\left(\|\boldsymbol{v}\|_{L^{2}\left(\Omega_{P}\right)^{d}}^{2}+\|\operatorname{div} \boldsymbol{v}\|_{L^{2}(\Omega)}^{2}\right)^{\frac{1}{2}}\|\operatorname{grad} \chi\|_{L^{2}\left(\Omega_{F}\right)^{d}}
$$

2) On the other hand, since $\boldsymbol{\varphi} \times \boldsymbol{n}$ vanishes on $\Gamma$, we have

$$
\int_{\Omega_{F}} \operatorname{curl} \varphi \cdot \boldsymbol{v} \mathrm{d} \boldsymbol{x}=\int_{\Omega_{F}} \operatorname{curl} \boldsymbol{v} \cdot \boldsymbol{\varphi} \mathrm{d} \boldsymbol{x} \leq\|\operatorname{curl} \boldsymbol{v}\|_{L^{2}\left(\Omega_{F}\right)^{\frac{d(d-1)}{2}}\|\varphi\|_{L^{2}\left(\Omega_{F}\right)} \frac{d(d-1)}{2}} .
$$

Using a generalized Poincaré-Friedrichs inequality which is proven in ([3], Cor. 3.19), we derive

$$
\int_{\Omega_{F}} \operatorname{curl} \varphi \cdot \boldsymbol{v} \mathrm{d} \boldsymbol{x} \leq\|\operatorname{curl} \boldsymbol{v}\|_{L^{2}\left(\Omega_{F}\right)} \frac{d(d-1)}{2}\|\operatorname{curl} \varphi\|_{L^{2}\left(\Omega_{F}\right)^{d}} .
$$

Inserting (2.11) and (2.12) into (2.9) and using (2.10) yield the desired property.

By combining (2.7) and Lemma 2.2, we obtain the ellipticity property

$$
\forall \boldsymbol{v} \in V, \quad a(\boldsymbol{v}, \boldsymbol{v}) \geq \frac{\inf \{\mu, \nu\}}{1+\alpha_{0}^{2}}\|\boldsymbol{v}\|_{X}^{2} .
$$

Moreover, since $H_{0}^{1}(\Omega)^{d}$ is continuously imbedded in $X$, it follows from the standard inf-sup condition for the Stokes problem ([18], Chap. I, Cor. 2.4), that there exists a constant $\beta>0$ such that

$$
\forall q \in L_{0}^{2}(\Omega), \quad \sup _{\boldsymbol{v} \in X} \frac{b(\boldsymbol{v}, q)}{\|\boldsymbol{v}\|_{X}} \geq \beta\|q\|_{L^{2}(\Omega)} .
$$

So the well-posedness of problem (2.5) follows from the standard theory of saddle-point problems. 
Theorem 2.3. For any data $\boldsymbol{f}$ in $L^{2}(\Omega)^{d}$, problem (2.5) has a unique solution $(\boldsymbol{u}, p)$ in $X \times L_{0}^{2}(\Omega)$. Moreover, this solution satisfies

$$
\|\boldsymbol{u}\|_{X}+\|p\|_{L^{2}(\Omega)} \leq c\|\boldsymbol{f}\|_{L^{2}(\Omega)^{d}}
$$

As a conclusion, it must be noted that standard arguments fail for proving a further regularity of the solution $(\boldsymbol{u}, p)$ on the whole domain.

\section{Another Formulation}

In view of the finite element discretization and according to the ideas in $[2,14,15,27]$, we now propose a modified formulation of problem (2.5), which relies on the introduction of a new unknown: the vorticity $\boldsymbol{\omega}$ associated with $\boldsymbol{u}$ in $\Omega_{F}$.

We denote by $\tilde{H}_{0}\left(\mathbf{c u r l}, \Omega_{F}\right)$ the space of functions in $L^{2}\left(\Omega_{F}\right)^{\frac{d(d-1)}{2}}$ with curl in $L^{2}\left(\Omega_{F}\right)^{d}$ and zero traces on $\Gamma$ in dimension $d=2$, zero tangential traces on $\Gamma$ in dimension $d=3$, provided with the graph norm $\|\cdot\|_{\tilde{H}\left(\mathbf{c u r l}, \Omega_{F}\right)}$ of the curl operator. Note that this space is rather different according to the dimension: it is equal to $H_{0}^{1}\left(\Omega_{F}\right)$ in dimension $d=2$ while, in dimension $d=3$, it coincides with the subspace of functions in $H\left(\right.$ curl, $\left.\Omega_{F}\right)$ (introduced in Sect. 2) with zero tangential traces on $\Gamma$. We consider the following problem:

Find $(\boldsymbol{\omega}, \boldsymbol{u}, p)$ in $\tilde{H}_{0}\left(\mathbf{c u r l}, \Omega_{F}\right) \times H_{0}(\operatorname{div}, \Omega) \times L_{0}^{2}(\Omega)$ such that

$$
\begin{aligned}
& \forall \boldsymbol{\varphi} \in \tilde{H}_{0}\left(\operatorname{curl}, \Omega_{F}\right), \quad \int_{\Omega_{F}} \boldsymbol{\omega} \cdot \boldsymbol{\varphi} \mathrm{d} \boldsymbol{x}-\int_{\Omega_{F}} \boldsymbol{u} \cdot \operatorname{curl} \boldsymbol{\varphi} \mathrm{d} \boldsymbol{x}=0, \\
& \forall \boldsymbol{v} \in H_{0}(\operatorname{div}, \Omega), \quad \mu \int_{\Omega_{P}} \boldsymbol{u} \cdot \boldsymbol{v} \mathrm{d} \boldsymbol{x}+\nu \int_{\Omega_{F}} \operatorname{curl} \boldsymbol{\omega} \cdot \boldsymbol{v} \mathrm{d} \boldsymbol{x}+b(\boldsymbol{v}, p)=\int_{\Omega} \boldsymbol{f} \cdot \boldsymbol{v} \mathrm{d} \boldsymbol{x}, \\
& \forall q \in L_{0}^{2}(\Omega), \quad b(\boldsymbol{u}, q)=0 .
\end{aligned}
$$

Remark 3.1. In opposite to the result investigated in Remark 2.1, problems (2.1) and (3.1) are fully equivalent whenever $p_{\mid \Omega_{F}}$ belongs $H^{1}\left(\Omega_{F}\right)$. This comes from the density of $\mathscr{D}(\Omega)^{d}$ in $H_{0}(\operatorname{div}, \Omega)$ and of $\mathscr{D}\left(\Omega_{F}\right)^{\frac{d(d-1)}{2}}$ in $\tilde{H}_{0}\left(\operatorname{curl}, \Omega_{F}\right)$, see ([18], Chap. I, Thms. 2.6 and 2.12) or ([28], Chap. 1, Thm. 1.3).

We first check the following property.

Proposition 3.2. For any solution $(\boldsymbol{\omega}, \boldsymbol{u}, p)$ of problem $(3.1)$ in the space $\tilde{H}_{0}\left(\mathbf{c u r l}, \Omega_{F}\right) \times H_{0}(\operatorname{div}, \Omega) \times L_{0}^{2}(\Omega)$, the pair $(\boldsymbol{u}, p)$ belongs to $X \times L_{0}^{2}(\Omega)$ and is a solution of problem (2.5).

Proof. The first line in problem (3.1) implies that curl $\boldsymbol{u}$ coincides with $\boldsymbol{\omega}$ in the distribution sense on $\Omega_{F}$, hence in $L^{2}\left(\Omega_{F}\right)^{\frac{d(d-1)}{2}}$. So the function $\boldsymbol{u}$ belongs to $X$. Taking $\boldsymbol{v}$ in $X$ in the second line of problem (3.1), integrating by parts and taking into account the fact that $\boldsymbol{\omega} \times \boldsymbol{n}$ vanishes on $\Gamma$ yields the first line in problem (2.5). Finally the last line in problem (3.1) is the same as the second line in problem (2.5).

To go further, we must prove that problem (3.1) admits a solution. So we first introduce the modified kernel

$$
W=\left\{\boldsymbol{v} \in H_{0}(\operatorname{div}, \Omega) ; \forall q \in L_{0}^{2}(\Omega), b(\boldsymbol{v}, q)=0\right\}=\left\{\boldsymbol{v} \in H_{0}(\operatorname{div}, \Omega) ; \operatorname{div} \boldsymbol{v}=0 \text { in } \Omega\right\},
$$

and the product space $\mathcal{X}=\tilde{H}_{0}\left(\operatorname{curl}, \Omega_{F}\right) \times W$. We also consider the kernel

$$
\mathcal{W}=\left\{(\boldsymbol{\theta}, \boldsymbol{v}) \in \mathcal{X} ; \quad \forall \boldsymbol{\varphi} \in \tilde{H}_{0}\left(\operatorname{curl}, \Omega_{F}\right), \quad \int_{\Omega_{F}} \boldsymbol{\theta} \cdot \boldsymbol{\varphi} \mathrm{d} \boldsymbol{x}-\int_{\Omega_{F}} \boldsymbol{v} \cdot \operatorname{curl} \boldsymbol{\varphi} \mathrm{d} \boldsymbol{x}=0\right\} .
$$

Due to the continuity of the bilinear form

$$
\mathcal{B}(V, \boldsymbol{\varphi})=\int_{\Omega_{F}} \boldsymbol{\theta} \cdot \boldsymbol{\varphi} \mathrm{d} \boldsymbol{x}-\int_{\Omega_{F}} \boldsymbol{v} \cdot \operatorname{curl} \boldsymbol{\varphi} \mathrm{d} \boldsymbol{x}, \quad \text { with } \quad V=(\boldsymbol{\theta}, \boldsymbol{v})
$$


on $\mathcal{X} \times \tilde{H}_{0}\left(\mathbf{c u r l}, \Omega_{F}\right), \mathcal{W}$ is a closed subspace of $\mathcal{X}$, hence is a Hilbert space. Moreover it can be checked that, thanks to the density of $\mathscr{D}\left(\Omega_{F}\right)^{\frac{d(d-1)}{2}}$ in $\tilde{H}_{0}\left(\mathbf{c u r l}, \Omega_{F}\right)$ quoted in Remark 3.1, that this space is equivalently defined by

$$
\mathcal{W}=\left\{V=(\boldsymbol{\theta}, \boldsymbol{v}) \in \tilde{H}_{0}\left(\operatorname{curl}, \Omega_{F}\right) \times W ; \boldsymbol{\theta}=\operatorname{curl} \boldsymbol{v} \text { in } \Omega_{F}\right\} .
$$

We consider the reduced problem

Find $U$ in $\mathcal{W}$ such that

$$
\forall \boldsymbol{v} \in W, \quad \mathcal{A}(U, \boldsymbol{v})=\int_{\Omega} \boldsymbol{f} \cdot \boldsymbol{v} \mathrm{d} \boldsymbol{x},
$$

where the bilinear form $\mathcal{A}(\cdot, \cdot)$ is now defined on $\left(\tilde{H}_{0}\left(\operatorname{curl}, \Omega_{F}\right) \times H_{0}(\operatorname{div}, \Omega)\right) \times H_{0}(\operatorname{div}, \Omega)$ by

$$
\mathcal{A}(U, \boldsymbol{v})=\mu \int_{\Omega_{P}} \boldsymbol{u} \cdot \boldsymbol{v} \mathrm{d} \boldsymbol{x}+\nu \int_{\Omega_{F}} \operatorname{curl} \boldsymbol{\omega} \cdot \boldsymbol{v} \mathrm{d} \boldsymbol{x}, \quad \text { with } \quad U=(\boldsymbol{\omega}, \boldsymbol{u}) .
$$

The analysis of problem (3.4) requires the following lemmas.

Lemma 3.3. The operator $C$ :

$$
\varphi \mapsto C \varphi= \begin{cases}\operatorname{curl} \varphi & \text { in } \Omega_{F}, \\ 0 & \text { in } \Omega_{P},\end{cases}
$$

is continuous from $\tilde{H}_{0}\left(\mathbf{c u r l}, \Omega_{F}\right)$ into $W$.

Proof. For any $\varphi$ in $\tilde{H}_{0}\left(\operatorname{curl}, \Omega_{F}\right)$, the function $\operatorname{curl} \varphi$ belongs to $L^{2}\left(\Omega_{F}\right)^{\frac{d(d-1)}{2}}$ and is divergence-free in $\Omega_{F}$, so it belongs to $H\left(\operatorname{div}, \Omega_{F}\right)$. Moreover, since $\boldsymbol{\varphi} \times \boldsymbol{n}$ vanishes on $\Gamma$, the same property holds for $\operatorname{curl} \boldsymbol{\varphi} \cdot \boldsymbol{n}$, so that $\operatorname{curl} \varphi$ belongs to $H_{0}\left(\operatorname{div}, \Omega_{F}\right)$. Since the extension by zero is continuous from this space into $H_{0}(\operatorname{div}, \Omega)$, the lemma is proved.

Lemma 3.4. The form $\mathcal{A}(\cdot, \cdot)$ satisfies the positivity property

$$
\forall \boldsymbol{v} \in W, \boldsymbol{v} \neq \mathbf{0}, \quad \sup _{U \in \mathcal{W}} \mathcal{A}(U, \boldsymbol{v})>0,
$$

and the inf-sup condition, for a constant $\gamma>0$,

$$
\forall U \in \mathcal{W}, \quad \sup _{\boldsymbol{v} \in W} \frac{\mathcal{A}(U, \boldsymbol{v})}{\|\boldsymbol{v}\|_{H(\operatorname{div}, \Omega)}} \geq \gamma\|U\|_{\mathcal{X}}
$$

Proof. We prove successively the two inequalities.

1) Let $\boldsymbol{v}$ be an element of $W$ such that $\mathcal{A}(U, \boldsymbol{v})$ vanishes for all $U$ in $\mathcal{W}$. Since $\boldsymbol{v} \cdot \boldsymbol{n}$ has a null integral on $\Gamma$, the problem

$$
\begin{cases}-\Delta \chi=0 & \text { in } \Omega_{F} \\ \partial_{n} \chi=\boldsymbol{v} \cdot \boldsymbol{n} & \text { on } \Gamma\end{cases}
$$

has a unique solution $\chi$ in $H^{1}\left(\Omega_{F}\right) \cap L_{0}^{2}\left(\Omega_{F}\right)$. Then the pair $U=(\boldsymbol{\omega}, \boldsymbol{u})$ defined by

$$
\boldsymbol{u}=\left\{\begin{array}{ll}
\boldsymbol{v} & \text { in } \Omega_{P}, \\
\operatorname{grad} \chi & \text { in } \Omega_{F},
\end{array} \quad \boldsymbol{\omega}=\mathbf{0},\right.
$$

belongs to $\mathcal{W}$ and the equation $\mathcal{A}(U, \boldsymbol{v})=0$ implies that $\boldsymbol{v}$ is zero on $\Omega_{P}$. Next, since $\boldsymbol{v}$ is divergence-free in $\Omega_{F}$, has a null normal trace on $\Gamma$ from the previous result and since $\Omega_{F}$ is simply-connected, there exists ([3], Thm. 3.17), a $\varphi$ in $\tilde{H}_{0}\left(\mathbf{c u r l}, \Omega_{F}\right)$ which is divergence-free and such that $\boldsymbol{v}$ is equal to $\operatorname{curl} \varphi$ on $\Omega_{F}$. Moreover, 
since $\varphi$ is divergence-free on $\Omega_{F}$ and $\Gamma$ is connected, there exists ([3], Thm. 3.12), a divergence-free $\boldsymbol{\psi}$ with zero normal trace on $\Gamma$ such that $\boldsymbol{\varphi}=\operatorname{curl} \boldsymbol{\psi}$. So the pair $U=(\boldsymbol{\omega}, \boldsymbol{u})$ defined by

$$
\boldsymbol{u}=\left\{\begin{array}{ll}
\mathbf{0} & \text { in } \Omega_{P}, \\
\boldsymbol{\psi} & \text { in } \Omega_{F},
\end{array} \quad \boldsymbol{\omega}=\boldsymbol{\varphi},\right.
$$

belongs to $\mathcal{W}$ and the equation $\mathcal{A}(U, \boldsymbol{v})=0$ implies that $\boldsymbol{v}$ is also zero on $\Omega_{F}$. This proves (3.7).

2) For any $U=(\boldsymbol{\omega}, \boldsymbol{u})$ in $\mathcal{W}$, when setting $\boldsymbol{v}$ equal to $\boldsymbol{u}+C \boldsymbol{\omega}$, we have

$$
\mathcal{A}(U, \boldsymbol{v})=\mu\|\boldsymbol{u}\|_{L^{2}\left(\Omega_{P}\right)^{d}}^{2}+\nu \int_{\Omega_{F}} \operatorname{curl} \boldsymbol{\omega} \cdot \boldsymbol{u} \mathrm{d} \boldsymbol{x}+\nu\|\operatorname{curl} \boldsymbol{\omega}\|_{L^{2}\left(\Omega_{F}\right)^{d}}^{2} .
$$

Integrating by parts int the middle term and recalling from (3.3) that $\boldsymbol{\omega}$ is equal to $\operatorname{curl} \boldsymbol{u}$, we obtain

$$
\begin{aligned}
\mathcal{A}(U, \boldsymbol{v}) \geq \min & \left\{\mu, \frac{\nu}{2}\right\} \\
& \left(\|\boldsymbol{u}\|_{L^{2}\left(\Omega_{P}\right)^{d}}^{2}+\|\operatorname{curl} \boldsymbol{u}\|_{L^{2}\left(\Omega_{F}\right)^{\frac{d(d-1)}{2}}}^{2}+\|\boldsymbol{\omega}\|_{L^{2}\left(\Omega_{F}\right)^{\frac{d(d-1)}{2}}}^{2}+\|\operatorname{curl} \boldsymbol{\omega}\|_{L^{2}\left(\Omega_{F}\right)^{d}}^{2}\right) .
\end{aligned}
$$

Thus, since $\boldsymbol{u}$ is divergence-free, it follows from Lemma 2.2 that

$$
\mathcal{A}(U, \boldsymbol{v}) \geq c\|U\|_{\mathcal{X}}^{2} .
$$

On the other hand, Lemma 3.3 yields that

$$
\|\boldsymbol{v}\|_{H(\operatorname{div}, \Omega)} \leq c\|U\|_{\mathcal{X}} .
$$

Combining the previous two inequalities leads to condition (3.8).

Standard arguments ([18], Chap. I, Lem. 4.1) (see also [5, 25] or [6], Thm. 2.1 and Rem. 2.1) allow for deriving from Lemma 3.4 the following result.

Proposition 3.5. For any data $\boldsymbol{f}$ in $L^{2}(\Omega)^{d}$, problem (3.4) has a unique solution $U=(\boldsymbol{\omega}, \boldsymbol{u})$ in $\mathcal{W}$.

Finally we introduce the space

$$
\mathcal{Y}=\left\{(\boldsymbol{\theta}, \boldsymbol{v}) \in \tilde{H}_{0}\left(\operatorname{curl}, \Omega_{F}\right) \times H_{0}(\operatorname{div}, \Omega) ; \quad \forall \boldsymbol{\varphi} \in \tilde{H}_{0}\left(\operatorname{curl}, \Omega_{F}\right), \quad \int_{\Omega_{F}} \boldsymbol{\theta} \cdot \boldsymbol{\varphi} \mathrm{d} \boldsymbol{x}-\int_{\Omega_{F}} \boldsymbol{v} \cdot \operatorname{curl} \boldsymbol{\varphi} \mathrm{d} \boldsymbol{x}=0\right\},
$$

and we consider the problem

Find $(U, p)$ in $\mathcal{Y} \times L_{0}^{2}(\Omega)$, with $U=(\boldsymbol{\omega}, \boldsymbol{u})$, such that

$$
\begin{aligned}
& \forall \boldsymbol{v} \in H_{0}(\operatorname{div}, \Omega), \quad \mathcal{A}(U, \boldsymbol{v})+b(\boldsymbol{v}, p)=\int_{\Omega} \boldsymbol{f} \cdot \boldsymbol{v} \mathrm{d} \boldsymbol{x}, \\
& \forall q \in L_{0}^{2}(\Omega), \quad b(\boldsymbol{u}, q)=0,
\end{aligned}
$$

for the form $b(\cdot, \cdot)$ defined in (2.4). Note that, since $X$ is imbedded in $H_{0}(\operatorname{div}, \Omega)$, the following inf-sup condition is obviously derived from (2.13):

$$
\forall q \in L_{0}^{2}(\Omega), \quad \sup _{\boldsymbol{v} \in H_{0}(\operatorname{div}, \Omega)} \frac{b(\boldsymbol{v}, q)}{\|\boldsymbol{v}\|_{H(\operatorname{div}, \Omega)}} \geq \beta\|q\|_{L^{2}(\Omega)} .
$$


This yields the following proposition.

Proposition 3.6. For any data $\boldsymbol{f}$ in $L^{2}(\Omega)^{d}$, problem (3.9) has a unique solution $(U, p)$ in $\mathcal{Y} \times L_{0}^{2}(\Omega)$. Moreover, the part $U$ of this solution belongs to $\mathcal{W}$ and is the unique solution of problem (3.4).

Thanks to the definition of the space $\mathcal{Y}$, problem (3.9) coincides with problem (3.1). So we now state the final result.

Corollary 3.7. For any data $\boldsymbol{f}$ in $L^{2}(\Omega)^{d}$, problem (3.1) has a unique solution $(\boldsymbol{\omega}, \boldsymbol{u}, p)$ in $\tilde{H}_{0}\left(\mathbf{c u r l}, \Omega_{F}\right) \times$ $H_{0}(\operatorname{div}, \Omega) \times L_{0}^{2}(\Omega)$. Moreover, the part $\boldsymbol{\omega}$ of this solution is equal to $\operatorname{curl} \boldsymbol{u}$ on $\Omega_{F}$.

Remark 3.8. Thanks to the two inf-sup conditions (3.8) and (3.10), the solution ( $\boldsymbol{\omega}, \boldsymbol{u}, p)$ of problem (3.1) satisfies the estimate

$$
\|\boldsymbol{\omega}\|_{\tilde{H}\left(\mathbf{c u r l}, \Omega_{F}\right)}+\|\boldsymbol{u}\|_{H(\operatorname{div}, \Omega)}+\|p\|_{L^{2}(\Omega)} \leq c\|\boldsymbol{f}\|_{L^{2}(\Omega)^{d}} .
$$

Moreover, the existence result stated in Corollary 3.7 and the previous estimate extend to slightly more general data $\boldsymbol{f}$, namely in the dual space of $H_{0}(\operatorname{div}, \Omega)$. However we have no direct applications for that.

Using formulation (3.1) as a basis for the discretization of problem (2.1) by the Galerkin method has two advantages:

- when replacing $H_{0}(\operatorname{div}, \Omega), L_{0}^{2}(\Omega)$ and $\tilde{H}_{0}\left(\mathbf{c u r l}, \Omega_{F}\right)$ by finite-dimensional subspaces, problem (3.1) results into a square linear system;

- the discrete spaces on $\Omega$ and on $\Omega_{F}$ can a priori be constructed in a completely independent way.

\section{Finite element discretization}

In what follows and for simplicity, we make the further assumption that both $\Omega$ and $\Omega_{F}$ are polygons in dimension $d=2$, polyhedra in dimension $d=3$. We introduce a regular family $\left(\mathcal{T}_{h}\right)_{h}$ of triangulations by closed triangles $(d=2)$ or tetrahedra $(d=3)$, in the usual sense that

- for each $h, \bar{\Omega}$ is the union of all elements of $\mathcal{T}_{h}$;

- for each $h$, the intersection of two different elements of $\mathcal{T}_{h}$, if not empty, is a corner, a whole edge or a whole face of both of them;

- the ratio of the diameter $h_{K}$ of an element $K$ in $\mathcal{T}_{h}$ to the diameter of its inscribed circle or sphere is bounded by a constant independent of $K$ and $h$;

and with the further condition

- for each $h$, the intersection of $\Gamma$ with the interior of any element $K$ of $\mathcal{T}_{h}$ is empty.

As usual, $h$ denotes the maximum of the diameters of the elements of $\mathcal{T}_{h}$. We denote by $\mathcal{T}_{h}^{F}$ the set of elements $K$ of $\mathcal{T}_{h}$ with are contained in $\bar{\Omega}_{F}$.

Next, for each $K$ in $\mathcal{T}_{h}$, we introduce the space $\mathcal{P}_{0}(K)$ of restrictions to $K$ of constant functions on $\mathbb{R}^{d}$, the space $\mathcal{P}_{1}(K)$ of restrictions to $K$ of affine functions on $\mathbb{R}^{d}$, the space $P_{K}$ of restrictions to $K$ of polynomials $\boldsymbol{p}$ of the form

$$
\boldsymbol{p}(\boldsymbol{x})=\boldsymbol{a}+b \boldsymbol{x}, \quad \boldsymbol{a} \in \mathbb{R}^{d}, \quad b \in \mathbb{R},
$$

and finally, in dimension $d=3$, the space $P_{K}^{\prime}$ of restrictions to $K$ of polynomials $\boldsymbol{p}$ of the form

$$
\boldsymbol{p}(\boldsymbol{x})=\boldsymbol{a}+\boldsymbol{b} \times \boldsymbol{x}, \quad \boldsymbol{a} \in \mathbb{R}^{3}, \quad \boldsymbol{b} \in \mathbb{R}^{3} .
$$

The spaces $P_{K}$ and $P_{K}^{\prime}$ and the corresponding finite elements are studied in ([24], Sect. 1.1). Next, we introduce the discrete spaces

$$
\begin{aligned}
\mathbb{D}_{h}(\Omega) & =\left\{\boldsymbol{v}_{h} \in H_{0}(\operatorname{div}, \Omega) ; \forall K \in \mathcal{T}_{h}, \boldsymbol{v}_{h \mid K} \in P_{K}\right\}, \\
\mathbb{M}_{h}(\Omega) & =\left\{q_{h} \in L_{0}^{2}(\Omega) ; \forall K \in \mathcal{T}_{h}, q_{h \mid K} \in \mathcal{P}_{0}(K)\right\} .
\end{aligned}
$$


Note that the finite element involved in the definition of the space $\mathbb{D}_{h}(\Omega)$ is that of Raviart and Thomas [26]. On the other hand, since the space $\tilde{H}_{0}\left(\operatorname{curl}, \Omega_{F}\right)$ is made of scalar functions in dimension $d=2$, of vector fields in dimension $d=3$, we use different discrete spaces to approximate it according to the dimension, namely

$$
\mathbb{C}_{h}\left(\Omega_{F}\right)= \begin{cases}\left\{\varphi_{h} \in H_{0}^{1}\left(\Omega_{F}\right) ; \forall K \in \mathcal{T}_{h}^{F}, \varphi_{h \mid K} \in \mathcal{P}_{1}(K)\right\}, & \text { if } d=2, \\ \left\{\varphi_{h} \in \tilde{H}_{0}\left(\operatorname{curl}, \Omega_{F}\right) ; \forall K \in \mathcal{T}_{h}^{F}, \varphi_{h \mid K} \in P_{K}^{\prime}\right\}, & \text { if } d=3 .\end{cases}
$$

The discrete problem is now derived from problem (3.1) by the Galerkin method. It reads

Find $\left(\boldsymbol{\omega}_{h}, \boldsymbol{u}_{h}, p_{h}\right)$ in $\mathbb{C}_{h}\left(\Omega_{F}\right) \times \mathbb{D}_{h}(\Omega) \times \mathbb{M}_{h}(\Omega)$ such that

$$
\begin{aligned}
& \forall \boldsymbol{\varphi}_{h} \in \mathbb{C}_{h}\left(\Omega_{F}\right), \quad \int_{\Omega_{F}} \boldsymbol{\omega}_{h} \cdot \boldsymbol{\varphi}_{h} \mathrm{~d} \boldsymbol{x}-\int_{\Omega_{F}} \boldsymbol{u}_{h} \cdot \operatorname{curl} \boldsymbol{\varphi}_{h} \mathrm{~d} \boldsymbol{x}=0, \\
& \forall \boldsymbol{v}_{h} \in \mathbb{D}_{h}(\Omega), \quad \mu \int_{\Omega_{P}} \boldsymbol{u}_{h} \cdot \boldsymbol{v}_{h} \mathrm{~d} \boldsymbol{x}+\nu \int_{\Omega_{F}} \operatorname{curl} \boldsymbol{\omega}_{h} \cdot \boldsymbol{v}_{h} \mathrm{~d} \boldsymbol{x}+b\left(\boldsymbol{v}_{h}, p_{h}\right)=\int_{\Omega} \boldsymbol{f} \cdot \boldsymbol{v}_{h} \mathrm{~d} \boldsymbol{x}, \\
& \forall q_{h} \in \mathbb{M}_{h}(\Omega), \quad b\left(\boldsymbol{u}_{h}, q_{h}\right)=0 .
\end{aligned}
$$

Note that, as scheduled at the end of the previous section, problem (4.3) results into a square linear system. Moreover the discretization is conforming.

Proving the existence of a solution is simpler than for the continuous problem. We introduce the kernel

$$
W_{h}=\left\{\boldsymbol{v}_{h} \in \mathbb{D}_{h}(\Omega) ; \forall q_{h} \in \mathbb{M}_{h}(\Omega), b\left(\boldsymbol{v}_{h}, q_{h}\right)=0\right\}
$$

and observe that, since the range of $\mathbb{D}_{h}(\Omega)$ by the divergence operator is contained in $\mathbb{M}_{h}(\Omega)$,

$$
W_{h}=\left\{\boldsymbol{v}_{h} \in \mathbb{D}_{h}(\Omega) ; \operatorname{div} \boldsymbol{v}_{h}=0 \text { in } \Omega\right\} .
$$

Proposition 4.1. For any data $\boldsymbol{f}$ in $L^{2}(\Omega)^{d}$, problem (4.3) has a unique solution in $\mathbb{C}_{h}\left(\Omega_{F}\right) \times \mathbb{D}_{h}(\Omega) \times \mathbb{M}_{h}(\Omega)$.

Proof. Since problem (4.3) results into a square linear system, it suffices to check that its only solution for $\boldsymbol{f}$ equal to zero is zero. In order to prove this, we take $\boldsymbol{f}$ equal to zero and choose $\boldsymbol{v}_{h}$ in the second equation of (4.3) equal to $\boldsymbol{u}_{h}$. Since it belongs to $W_{h}$, this yields

$$
\mu\left\|\boldsymbol{u}_{h}\right\|_{L^{2}\left(\Omega_{P}\right)^{d}}^{2}+\nu \int_{\Omega_{F}} \operatorname{curl} \omega_{h} \cdot \boldsymbol{u}_{h} \mathrm{~d} \boldsymbol{x}=0
$$

Then, using the first line of $(4.3)$ with $\varphi_{h}$ equal to $\omega_{h}$ leads to

$$
\mu\left\|\boldsymbol{u}_{h}\right\|_{L^{2}\left(\Omega_{P}\right)^{d}}^{2}+\nu\left\|\boldsymbol{\omega}_{h}\right\|_{L^{2}\left(\Omega_{F}\right)}^{2} \frac{d(d-1)}{2}=0
$$

so that $\boldsymbol{u}_{h}$ is zero on $\Omega_{P}$ and $\boldsymbol{\omega}_{h}$ is zero on $\Omega_{F}$. Thus, we have the equation

$$
\forall \varphi_{h} \in \mathbb{C}_{h}\left(\Omega_{F}\right), \quad \int_{\Omega_{F}} \boldsymbol{u}_{h} \cdot \operatorname{curl} \boldsymbol{\varphi}_{h} \mathrm{~d} \boldsymbol{x}=0
$$

On the other hand, it can be checked by similar arguments as in [19] that any function which is the restriction to $\Omega_{F}$ of a function in $W_{h}$ and moreover has a null normal trace on $\Gamma$ is the curl of a function $\tilde{\varphi}_{h}$ in $\mathbb{C}_{h}\left(\Omega_{F}\right)$. So the previous equation implies that $\boldsymbol{u}_{h}$ is zero on $\Omega_{F}$ also. Finally, the pressure $p_{h}$ satisfies

$$
\forall \boldsymbol{v}_{h} \in \mathbb{D}_{h}(\Omega), \quad b\left(\boldsymbol{v}_{h}, p_{h}\right)=0,
$$


and it follows from $[26]$ that $p_{h}$ is zero on $\Omega$. This concludes the proof.

We now intend to prove a priori error estimates between the solutions $(\boldsymbol{\omega}, \boldsymbol{u}, p)$ and $\left(\boldsymbol{\omega}_{h}, \boldsymbol{u}_{h}, p_{h}\right)$. In order to do this, we define the product space $\mathcal{X}_{h}=\mathbb{C}_{h}\left(\Omega_{F}\right) \times W_{h}$ and the kernel

$$
\mathcal{W}_{h}=\left\{\left(\boldsymbol{\theta}_{h}, \boldsymbol{v}_{h}\right) \in \mathcal{X}_{h} ; \quad \forall \boldsymbol{\varphi}_{h} \in \mathbb{C}_{h}\left(\Omega_{F}\right), \quad \int_{\Omega_{F}} \boldsymbol{\theta}_{h} \cdot \boldsymbol{\varphi}_{h} \mathrm{~d} \boldsymbol{x}-\int_{\Omega_{F}} \boldsymbol{v}_{h} \cdot \operatorname{curl} \boldsymbol{\varphi}_{h} \mathrm{~d} \boldsymbol{x}=0\right\} .
$$

We first consider the problem

Find $U_{h}$ in $\mathcal{W}_{h}$ such that

$$
\forall \boldsymbol{v}_{h} \in W_{h}, \quad \mathcal{A}\left(U_{h}, \boldsymbol{v}_{h}\right)=\int_{\Omega} \boldsymbol{f} \cdot \boldsymbol{v}_{h} \mathrm{~d} \boldsymbol{x} .
$$

Let $W_{h}^{F}$ denote the space of restrictions to $\Omega_{F}$ of functions $\boldsymbol{v}_{h}$ in $W_{h}$ which moreover have a zero normal trace on $\Gamma$. The following property is established in ([27], Sect. 2.1), and ([15], Prop. 5), in the case of dimension $d=2$. So we only prove it in dimension $d=3$.

Lemma 4.2. There exists a constant $\sigma_{0}$ independent of $h$ such that the following property holds

$$
\forall \boldsymbol{v}_{h} \in W_{h}^{F}, \quad \sup _{\boldsymbol{\varphi}_{h} \in \mathbb{C}_{h}\left(\Omega_{F}\right)} \frac{\int_{\Omega_{F}} \boldsymbol{v}_{h} \cdot \operatorname{curl} \boldsymbol{\varphi}_{h} \mathrm{~d} \boldsymbol{x}}{\left\|\boldsymbol{\varphi}_{h}\right\|_{H\left(\operatorname{curl}, \Omega_{F}\right)}} \geq \sigma_{0}\left\|\boldsymbol{v}_{h}\right\|_{L^{2}\left(\Omega_{F}\right)^{d}}
$$

Proof. In dimension $d=3$, we consider the problem

Find $\left(\psi_{h}, \lambda_{h}\right)$ in $\mathbb{C}_{h}\left(\Omega_{F}\right) \times \mathbb{H}_{h}\left(\Omega_{F}\right)$ such that

$$
\begin{aligned}
& \forall \boldsymbol{\varphi}_{h} \in \mathbb{C}_{h}\left(\Omega_{F}\right), \quad \int_{\Omega_{F}} \operatorname{curl} \boldsymbol{\psi}_{h} \cdot \operatorname{curl} \boldsymbol{\varphi}_{h} \mathrm{~d} \boldsymbol{x}+\int_{\Omega_{F}} \boldsymbol{\varphi}_{h} \cdot \operatorname{grad} \lambda_{h} \mathrm{~d} \boldsymbol{x}=\int_{\Omega_{F}} \boldsymbol{v}_{h} \cdot \operatorname{curl} \boldsymbol{\varphi}_{h} \mathrm{~d} \boldsymbol{x}, \\
& \forall \mu_{h} \in \mathbb{H}_{h}\left(\Omega_{F}\right), \quad \int_{\Omega_{F}} \boldsymbol{\psi}_{h} \cdot \operatorname{grad} \mu_{h} \mathrm{~d} \boldsymbol{x}=0,
\end{aligned}
$$

where $\mathbb{H}_{h}\left(\Omega_{F}\right)$ stands for the space of functions in $H_{0}^{1}\left(\Omega_{F}\right)$ such that their restrictions to any $K$ in $\mathcal{T}_{h}^{F}$ belong to $\mathcal{P}_{1}(K)$. It is proved in ([3], Props. 4.11 and 4.12) that, since $\Omega_{F}$ is simply-connected and has a connected boundary, this problem has a unique solution $\left(\psi_{h}, \lambda_{h}\right)$, that this $\lambda_{h}$ is equal to zero and moreover that

$$
\left\|\boldsymbol{\psi}_{h}\right\|_{\tilde{H}\left(\operatorname{curl}, \Omega_{F}\right)} \leq c\left\|\operatorname{curl} \boldsymbol{\psi}_{h}\right\|_{L^{2}\left(\Omega_{F}\right)^{3}}
$$

So taking $\varphi_{h}$ equal to $\boldsymbol{\psi}_{h}$ yields

$$
\sup _{\boldsymbol{\varphi}_{h} \in \mathbb{C}_{h}\left(\Omega_{F}\right)} \frac{\int_{\Omega_{F}} \boldsymbol{v}_{h} \cdot \operatorname{curl} \boldsymbol{\varphi}_{h} \mathrm{~d} \boldsymbol{x}}{\left\|\boldsymbol{\varphi}_{h}\right\|_{\tilde{H}\left(\operatorname{curl}, \Omega_{F}\right)}} \geq c\left\|\operatorname{curl} \boldsymbol{\psi}_{h}\right\|_{L^{2}\left(\Omega_{F}\right)^{3}} .
$$

On the other hand, it can be checked by similar arguments as in [19] loc. cit. that any function $\boldsymbol{v}_{h}$ in $W_{h}^{F}$ is the curl of a function $\tilde{\varphi}_{h}$ in $\mathbb{C}_{h}\left(\Omega_{F}\right)$. So taking $\boldsymbol{\varphi}_{h}$ equal to $\tilde{\varphi}_{h}$ in the previous problem leads to

$$
\left\|\boldsymbol{v}_{h}\right\|_{L^{2}\left(\Omega_{F}\right)^{3}} \leq\left\|\operatorname{curl} \boldsymbol{\psi}_{h}\right\|_{L^{2}\left(\Omega_{F}\right)^{3}}
$$

Combining the two last inequalities gives the desired result.

The extension to functions in $W_{h}$ which have non zero normal traces on $\Gamma$ is more technical. Let $h_{\min }^{\Gamma}$ stand for the smallest length of the edges $e(d=2)$ or diameter of the faces $e(d=3)$ of elements of $\mathcal{T}_{h}$ which are contained in $\Gamma$. 
Lemma 4.3. There exists a constant $\sigma$ independent of $h$ such that the following property holds

$$
\forall \boldsymbol{v}_{h} \in W_{h}, \quad\left\|\boldsymbol{v}_{h}\right\|_{L^{2}\left(\Omega_{P}\right)^{d}}+\sup _{\boldsymbol{\varphi}_{h} \in \mathbb{C}_{h}\left(\Omega_{F}\right)} \frac{\int_{\Omega_{F}} \boldsymbol{v}_{h} \cdot \operatorname{curl} \boldsymbol{\varphi}_{h} \mathrm{~d} \boldsymbol{x}}{\left\|\boldsymbol{\varphi}_{h}\right\|_{H\left(\operatorname{curl}, \Omega_{F}\right)}} \geq \sigma\left(h_{\min }^{\Gamma}\right)^{\frac{1}{2}}\left|\log h_{\min }^{\Gamma}\right|^{-1}\left\|\boldsymbol{v}_{h}\right\|_{L^{2}(\Omega)^{d}}
$$

Proof. Since the second term in the left-hand side of (4.7) is nonnegative, it suffices to prove the modified estimate, for some constant $\rho, 0<\rho \leq 1$,

$$
\forall \boldsymbol{v}_{h} \in W_{h}, \quad\left\|\boldsymbol{v}_{h}\right\|_{L^{2}\left(\Omega_{P}\right)^{d}}+\rho \sup _{\boldsymbol{\varphi}_{h} \in \mathbb{C}_{h}\left(\Omega_{F}\right)} \frac{\int_{\Omega_{F}} \boldsymbol{v}_{h} \cdot \operatorname{curl} \boldsymbol{\varphi}_{h} \mathrm{~d} \boldsymbol{x}}{\left\|\boldsymbol{\varphi}_{h}\right\|_{H\left(\operatorname{curl}, \Omega_{F}\right)}} \geq \sigma\left\|\boldsymbol{v}_{h}\right\|_{L^{2}(\Omega)^{d}} .
$$

Next, for any $\boldsymbol{v}_{h}$ in $W_{h}$, we consider the problem

Find $\tilde{\lambda}_{h}$ in $\tilde{\mathbb{H}}_{h}\left(\Omega_{F}\right) / \mathbb{R}$ such that

$$
\forall \tilde{\mu}_{h} \in \tilde{\mathbb{H}}_{h}\left(\Omega_{F}\right), \quad \sum_{K \in \mathcal{T}_{h}^{F}} \int_{K} \operatorname{grad} \tilde{\lambda}_{h} \cdot \operatorname{grad} \tilde{\mu}_{h} \mathrm{~d} \boldsymbol{x}=\int_{\Gamma} \boldsymbol{v}_{h} \cdot \boldsymbol{n} \tilde{\mu}_{h} \mathrm{~d} \boldsymbol{\tau},
$$

where $\tilde{\mathbb{H}}_{h}\left(\Omega_{F}\right)$ stands for the space of functions in $L^{2}\left(\Omega_{F}\right)$ such that their restriction to each $K$ in $\mathcal{T}_{h}^{F}$ belongs to $\mathcal{P}_{1}(K)$ and which are continuous in the midpoints of the edges $(d=2)$ or barycenters of the faces $(d=3)$ of all elements of $\mathcal{T}_{h}^{F}$ (the corresponding finite element was introduced in [10]). Since $\boldsymbol{v}_{h}$ is divergence-free in $\Omega_{F}$, the integral on $\Gamma$ of its normal trace vanishes, so that this problem has a unique solution $\tilde{\lambda}_{h}$. Next, for each edge $(d=2)$ or face $(d=3) e$ of an element of $\mathcal{T}_{h}^{F}$, taking $\tilde{\mu}_{h}$ equal to the basis function associated with the midpoint or barycenter of $e$ yields that

(i) if $e$ is the intersection of two elements of $\mathcal{T}_{h}^{F}$, the jump of $\frac{\partial \tilde{\lambda}_{h}}{\partial n}$ through $e$ vanishes;

(ii) if $e$ is contained in $\Gamma$, the normal trace $\frac{\partial \tilde{\lambda}_{h}}{\partial n}$ is equal to $\boldsymbol{v}_{h} \cdot \boldsymbol{n}$ on $e$.

As a consequence, the function grad $\tilde{\lambda}_{h}$ belongs to $H\left(\operatorname{div}, \Omega_{F}\right)$ and is divergence-free, so the function $\boldsymbol{v}_{h}^{0}=$ $\boldsymbol{v}_{h}-\operatorname{grad} \tilde{\lambda}_{h}$ belongs to $W_{h}^{F}$. Applying Lemma 4.2 to this function yields that

$$
\sup _{\boldsymbol{\varphi}_{h} \in \mathbb{C}_{h}\left(\Omega_{F}\right)} \frac{\int_{\Omega_{F}} \boldsymbol{v}_{h}^{0} \cdot \operatorname{curl} \boldsymbol{\varphi}_{h} \mathrm{~d} \boldsymbol{x}}{\left\|\boldsymbol{\varphi}_{h}\right\|_{H\left(\operatorname{curl}, \Omega_{F}\right)}} \geq \sigma_{0}\left\|\boldsymbol{v}_{h}^{0}\right\|_{L^{2}\left(\Omega_{F}\right)^{d}}
$$

so that

$$
\sup _{\boldsymbol{\varphi}_{h} \in \mathbb{C}_{h}\left(\Omega_{F}\right)} \frac{\int_{\Omega_{F}} \boldsymbol{v}_{h} \cdot \operatorname{curl} \boldsymbol{\varphi}_{h} \mathrm{~d} \boldsymbol{x}}{\left\|\boldsymbol{\varphi}_{h}\right\|_{H\left(\operatorname{curl}, \Omega_{F}\right)}} \geq \sigma_{0}\left\|\boldsymbol{v}_{h}\right\|_{L^{2}\left(\Omega_{F}\right)^{d}}-\left(1+\sigma_{0}\right)\left\|\operatorname{grad} \tilde{\lambda}_{h}\right\|_{L^{2}\left(\Omega_{F}\right)^{d}}
$$

In order to bound this last norm, we take $\tilde{\mu}_{h}$ equal to $\tilde{\lambda}_{h}$ in (4.9), so that

$$
\left\|\operatorname{grad} \tilde{\lambda}_{h}\right\|_{L^{2}\left(\Omega_{F}\right)^{d}}^{2} \leq \int_{\Gamma} \boldsymbol{v}_{h} \cdot \boldsymbol{n} \tilde{\lambda}_{h} \mathrm{~d} \boldsymbol{\tau} .
$$

Using inequality (A.9) of the appendix gives

$$
\int_{\Gamma} \boldsymbol{v}_{h} \cdot \boldsymbol{n} \tilde{\lambda}_{h} \mathrm{~d} \boldsymbol{\tau} \leq \alpha(h)\left\|\boldsymbol{v}_{h}\right\|_{L^{2}\left(\Omega_{P}\right)^{d}}\left(\sum_{K \in \mathcal{T}_{h}^{F}}\left\|\tilde{\lambda}_{h}\right\|_{H^{1}(K)}^{2}\right)^{\frac{1}{2}},
$$


with $\alpha(h)=\alpha^{*}\left(h_{\min }^{\Gamma}\right)^{-\frac{1}{2}}\left|\log h_{\min }^{\Gamma}\right|$. Moreover the function $\tilde{\lambda}_{h}$ is defined up to an additive constant, so it can be chosen to have a null mean value on $\Omega_{F}$. Thus using ([1], Lem. 4.2), yields

$$
\left(\sum_{K \in \mathcal{T}_{h}^{F}}\left\|\tilde{\lambda}_{h}\right\|_{H^{1}(K)}^{2}\right)^{\frac{1}{2}} \leq c_{0}\left\|\operatorname{grad} \tilde{\lambda}_{h}\right\|_{L^{2}\left(\Omega_{F}\right)^{d}}
$$

Inserting this into (4.11) gives

$$
\left\|\operatorname{grad} \tilde{\lambda}_{h}\right\|_{L^{2}\left(\Omega_{F}\right)^{d}} \leq c_{0} \alpha(h)\left\|\boldsymbol{v}_{h}\right\|_{L^{2}\left(\Omega_{P}\right)^{d}}
$$

whence, by (4.10),

$$
\sup _{\boldsymbol{\varphi}_{h} \in \mathbb{C}_{h}\left(\Omega_{F}\right)} \frac{\int_{\Omega_{F}} \boldsymbol{v}_{h} \cdot \operatorname{curl} \boldsymbol{\varphi}_{h} \mathrm{~d} \boldsymbol{x}}{\left\|\boldsymbol{\varphi}_{h}\right\|_{H\left(\operatorname{curl}, \Omega_{F}\right)}} \geq \sigma_{0}\left\|\boldsymbol{v}_{h}\right\|_{L^{2}\left(\Omega_{F}\right)^{d}}-\left(1+\sigma_{0}\right) c_{0} \alpha(h)\left\|\boldsymbol{v}_{h}\right\|_{L^{2}\left(\Omega_{P}\right)^{d}} .
$$

We now use (4.8) with $\rho=\left(2\left(1+\sigma_{0}\right) c_{0} \alpha(h)\right)^{-1}$ and we obtain the desired result.

Remark 4.4. Let us consider the function $\boldsymbol{v}_{h}$ in $\mathbb{D}_{h}(\Omega)$ which is constant equal to $\overline{\boldsymbol{v}}$ on $\Omega_{F}$ and satisfies, for all edges or faces $e$ of elements of $\mathcal{T}_{h} \backslash \mathcal{T}_{h}^{F}$,

$$
\int_{e} \boldsymbol{v}_{h} \cdot \boldsymbol{n} \mathrm{d} \boldsymbol{\tau}= \begin{cases}\int_{e} \overline{\boldsymbol{v}} \cdot \boldsymbol{n} \mathrm{d} \boldsymbol{\tau} & \text { if } e \text { is contained in } \Gamma \\ 0 & \text { otherwise. }\end{cases}
$$

Then, it can be checked than

and also that

$$
\forall \boldsymbol{\varphi}_{h} \in \mathbb{C}_{h}\left(\Omega_{F}\right), \quad \int_{\Omega_{F}} \boldsymbol{v}_{h} \cdot \operatorname{curl} \boldsymbol{\varphi}_{h} \mathrm{~d} \boldsymbol{x}=0
$$

$$
\left\|\boldsymbol{v}_{h}\right\|_{L^{2}(\Omega)^{d}} \geq|\overline{\boldsymbol{v}}| \operatorname{meas}\left(\Omega_{F}\right) \quad \text { and } \quad\left\|\boldsymbol{v}_{h}\right\|_{L^{2}\left(\Omega_{P}\right)^{d}} \leq c\left(h^{\Gamma}\right)^{\frac{1}{2}}|\overline{\boldsymbol{v}}|,
$$

where $h^{\Gamma}$ denotes the largest length of the edges $e$ or diameter of the faces $e$ of elements of $\mathcal{T}_{h}$ which are contained in $\Gamma$. Even if this function $\boldsymbol{v}_{h}$ does not belong to $W_{h}$, this seems to indicate that, at least when the family of triangulations $\left(\mathcal{T}_{h}\right)_{h}$ is uniformly regular in a neighbourhood of $\Gamma$ and up to the $\left|\log h_{\min }^{\Gamma}\right|^{-1}$, the constant in the right-hand side of (4.7) cannot be improved.

It is readily checked that the operator $C$ introduced in (3.6) maps $\mathbb{C}_{h}\left(\Omega_{F}\right)$ into $\mathbb{D}_{h}(\Omega)$ and even into $W_{h}$. So we are now in a position to prove the following inf-sup condition.

Lemma 4.5. The form $\mathcal{A}(\cdot, \cdot)$ satisfies the inf-sup condition, for a constant $\gamma^{*}>0$ independent of $h$,

$$
\forall U_{h} \in \mathcal{W}_{h}, \quad \sup _{\boldsymbol{v}_{h} \in W_{h}} \frac{\mathcal{A}\left(U_{h}, \boldsymbol{v}_{h}\right)}{\left\|\boldsymbol{v}_{h}\right\|_{H(\operatorname{div}, \Omega)}} \geq \gamma^{*}\left(h_{\min }^{\Gamma}\right)^{\frac{1}{2}}\left|\log h_{\min }^{\Gamma}\right|^{-1}\left\|U_{h}\right\|_{\mathcal{X}} .
$$

Proof. For any $U_{h}=\left(\boldsymbol{\omega}_{h}, \boldsymbol{u}_{h}\right)$ in $\mathcal{W}_{h}$, we take $\boldsymbol{v}_{h}=\boldsymbol{u}_{h}+C \boldsymbol{\omega}_{h}$. This gives

$$
\mathcal{A}\left(U_{h}, \boldsymbol{v}_{h}\right)=\mu\left\|\boldsymbol{u}_{h}\right\|_{L^{2}\left(\Omega_{P}\right)^{d}}^{2}+\nu \int_{\Omega_{F}} \operatorname{curl} \boldsymbol{\omega}_{h} \cdot \boldsymbol{u}_{h} \mathrm{~d} \boldsymbol{x}+\nu\left\|\operatorname{curl} \boldsymbol{\omega}_{h}\right\|_{L^{2}\left(\Omega_{F}\right)^{d}}^{2},
$$

whence, thanks to the definition of $\mathcal{W}_{h}$,

$$
\mathcal{A}\left(U_{h}, \boldsymbol{v}_{h}\right)=\mu\left\|\boldsymbol{u}_{h}\right\|_{L^{2}\left(\Omega_{P}\right)^{d}}^{2}+\nu\left\|\boldsymbol{\omega}_{h}\right\|_{L^{2}\left(\Omega_{F}\right)^{\frac{d(d-1)}{2}}}^{2}+\nu\left\|\operatorname{curl} \boldsymbol{\omega}_{h}\right\|_{L^{2}\left(\Omega_{F}\right)^{d}}^{2}
$$


On the other hand, we have

$$
\sup _{\boldsymbol{\varphi}_{h} \in \mathbb{C}_{h}\left(\Omega_{F}\right)} \frac{\int_{\Omega_{F}} \boldsymbol{u}_{h} \cdot \operatorname{curl} \boldsymbol{\varphi}_{h} \mathrm{~d} \boldsymbol{x}}{\left\|\boldsymbol{\varphi}_{h}\right\|_{\tilde{H}\left(\operatorname{curl}, \Omega_{F}\right)}} \leq \sup _{\boldsymbol{\varphi}_{h} \in \mathbb{C}_{h}\left(\Omega_{F}\right)} \frac{\int_{\Omega_{F}} \boldsymbol{\omega}_{h} \cdot \boldsymbol{\varphi}_{h} \mathrm{~d} \boldsymbol{x}}{\left\|\boldsymbol{\varphi}_{h}\right\|_{L^{2}\left(\Omega_{F}\right)^{\frac{d(d-1)}{2}}}} \leq\left\|\boldsymbol{\omega}_{h}\right\|_{L^{2}\left(\Omega_{F}\right)^{\frac{d(d-1)}{2}}} .
$$

Combining all this yields

$$
\begin{aligned}
\mathcal{A}\left(U_{h}, \boldsymbol{v}_{h}\right) \geq \min \left\{\mu, \frac{\nu}{2}\right\}\left(\left\|\boldsymbol{u}_{h}\right\|_{L^{2}\left(\Omega_{P}\right)^{d}}^{2}+\left(\sup _{\boldsymbol{\varphi}_{h} \in \mathbb{C}_{h}\left(\Omega_{F}\right)} \frac{\int_{\Omega_{F}} \boldsymbol{u}_{h} \cdot \operatorname{curl} \boldsymbol{\varphi}_{h} \mathrm{~d} \boldsymbol{x}}{\left\|\boldsymbol{\varphi}_{h}\right\|_{H\left(\operatorname{curl}, \Omega_{F}\right)}}\right)^{2}\right. & \left.+\left\|\boldsymbol{\omega}_{h}\right\|_{L^{2}\left(\Omega_{F}\right)^{\frac{d(d-1)}{2}}}^{2}+\left\|\operatorname{curl} \boldsymbol{\omega}_{h}\right\|_{L^{2}\left(\Omega_{F}\right)^{d}}^{2}\right) .
\end{aligned}
$$

Then applying Lemma 4.3 yields

$$
\mathcal{A}\left(U_{h}, \boldsymbol{v}_{h}\right) \geq \min \left\{\mu, \frac{\nu}{2}\right\} \sigma\left(h_{\min }^{\Gamma}\right)^{\frac{1}{2}}\left|\log h_{\min }^{\Gamma}\right|^{-1}\left\|U_{h}\right\|_{\mathcal{X}}^{2} .
$$

On the other hand, we derive from Lemma 3.3 that

$$
\left\|\boldsymbol{v}_{h}\right\|_{H(\operatorname{div}, \Omega)} \leq c\left\|U_{h}\right\|_{\mathcal{X}}
$$

whence the desired condition.

Obviously, if $\left(\boldsymbol{\omega}_{h}, \boldsymbol{u}_{h}, p_{h}\right)$ denotes the solution of problem $(4.3)$, the pair $U_{h}=\left(\boldsymbol{\omega}_{h}, \boldsymbol{u}_{h}\right)$ is a solution of problem (4.4). This yields for any $Z_{h}$ in $\mathcal{W}_{h}$ and $\boldsymbol{v}_{h}$ in $W_{h}$

$$
\mathcal{A}\left(U_{h}-Z_{h}, \boldsymbol{v}_{h}\right)=\int_{\Omega} \boldsymbol{f} \cdot \boldsymbol{v}_{h} \mathrm{~d} \boldsymbol{x}-\mathcal{A}\left(Z_{h}, \boldsymbol{v}_{h}\right) .
$$

Then, noting that $W_{h}$ is contained in $W$, we use (3.4), which gives

$$
\mathcal{A}\left(U_{h}-Z_{h}, \boldsymbol{v}_{h}\right)=\mathcal{A}\left(U-Z_{h}, \boldsymbol{v}_{h}\right)
$$

Applying Lemma 4.5 and a triangle inequality leads to the first estimate

$$
\left\|U-U_{h}\right\|_{\mathcal{X}} \leq c\left(h_{\min }^{\Gamma}\right)^{-\frac{1}{2}}\left|\log h_{\min }^{\Gamma}\right| \inf _{Z_{h} \in \mathcal{W}_{h}}\left\|U-Z_{h}\right\|_{\mathcal{X}}
$$

To go further, we note that $\mathcal{W}_{h}$ is the kernel of the form $\mathcal{B}(\cdot, \cdot)$ introduced in $(3.2)$.

Lemma 4.6. The form $\mathcal{B}(\cdot, \cdot)$ satisfies the inf-sup condition, for a constant $\delta>0$,

$$
\forall \boldsymbol{\varphi}_{h} \in \mathbb{C}_{h}\left(\Omega_{F}\right), \quad \sup _{V_{h} \in \mathcal{X}_{h}} \frac{\mathcal{B}\left(V_{h}, \varphi_{h}\right)}{\left\|V_{h}\right\| \mathcal{X}} \geq \delta\left\|\boldsymbol{\varphi}_{h}\right\|_{\tilde{H}\left(\operatorname{curl}, \Omega_{F}\right)} .
$$

Proof. With any $\boldsymbol{\varphi}_{h}$ in $\mathbb{C}_{h}\left(\Omega_{F}\right)$, we associate the pair $V_{h}=\left(\boldsymbol{\varphi}_{h},-C \boldsymbol{\varphi}_{h}\right)$, which belongs to $\mathcal{X}_{h}$. Thus, it is readily checked that

$$
\mathcal{B}\left(V_{h}, \varphi_{h}\right)=\left\|\boldsymbol{\varphi}_{h}\right\|_{\tilde{H}\left(\operatorname{curl}, \Omega_{F}\right)}^{2},
$$


and, from Lemma 3.3, that

$$
\left\|V_{h}\right\|_{\mathcal{X}} \leq c\left\|\boldsymbol{\varphi}_{h}\right\|_{\tilde{H}\left(\operatorname{curl}, \Omega_{F}\right)} .
$$

This gives the desired result.

By combining Lemma 4.6 with ([18], Chap. II, form. (1.16)) (see also [6], Prop. 2.1), we derive from (4.13) the second estimate

$$
\left\|U-U_{h}\right\|_{\mathcal{X}} \leq c\left(h_{\min }^{\Gamma}\right)^{-\frac{1}{2}}\left|\log h_{\min }^{\Gamma}\right| \inf _{Z_{h} \in \mathcal{X}_{h}}\left\|U-Z_{h}\right\|_{\mathcal{X}}
$$

Finally, the following inf-sup condition is proven in ([26], Thm. 4): for a constant $\beta^{*}>0$ independent of $h$,

$$
\forall q_{h} \in \mathbb{M}_{h}(\Omega), \quad \sup _{\boldsymbol{v}_{h} \in \mathbb{D}_{h}(\Omega)} \frac{b\left(\boldsymbol{v}_{h}, q_{h}\right)}{\left\|\boldsymbol{v}_{h}\right\|_{H(\operatorname{div}, \Omega)}} \geq \beta^{*}\left\|q_{h}\right\|_{L^{2}(\Omega)} .
$$

This allows first for replacing in (4.15)

$$
\inf _{Z_{h} \in \mathcal{X}_{h}}\left\|U-Z_{h}\right\| \mathcal{X} \leq c \inf _{Z_{h} \in \mathbb{C}_{h}\left(\Omega_{F}\right) \times \mathbb{D}_{h}(\Omega)}\left\|U-Z_{h}\right\|_{\tilde{H}\left(\operatorname{curl}, \Omega_{F}\right) \times H(\operatorname{div}, \Omega)},
$$

and also, by standard arguments that we omit here, for proving an estimate of the error on the pressure. Combining all this leads to

$$
\begin{aligned}
& \left\|\boldsymbol{\omega}-\boldsymbol{\omega}_{h}\right\|_{\tilde{H}\left(\operatorname{curl}, \Omega_{F}\right)}+\left\|\boldsymbol{u}-\boldsymbol{u}_{h}\right\|_{H(\operatorname{div}, \Omega)}+\left\|p-p_{h}\right\|_{L^{2}(\Omega)} \\
& \leq c\left(h_{\min }^{\Gamma}\right)^{-\frac{1}{2}}\left|\log h_{\min }^{\Gamma}\right|\left(\inf _{\boldsymbol{\theta}_{h} \in \mathbb{C}_{h}\left(\Omega_{F}\right)}\left\|\boldsymbol{\omega}-\boldsymbol{\theta}_{h}\right\|_{\tilde{H}\left(\operatorname{curl}, \Omega_{F}\right)}+\inf _{\boldsymbol{v}_{h} \in \mathbb{D}_{h}(\Omega)}\left\|\boldsymbol{u}-\boldsymbol{v}_{h}\right\|_{H(\operatorname{div}, \Omega)}+\inf _{q_{h} \in \mathbb{M}_{h}(\Omega)}\left\|p-q_{h}\right\|_{L^{2}(\Omega)}\right) .
\end{aligned}
$$

The approximation properties of the spaces $\mathbb{M}_{h}(\Omega)$ and $\mathbb{C}_{h}\left(\Omega_{F}\right)$ in the two-dimensional case are well-known while those of $\mathbb{D}_{h}(\Omega)$ and $\mathbb{C}_{h}\left(\Omega_{F}\right)$ for $d=3$ can be derived from ([24], Thms. 2 and 4 ) (see also [26]). This leads to the final a priori error estimate.

Theorem 4.7. Assume that the solution $(\boldsymbol{u}, p)$ of problem $(2.1)$ belongs to $H^{s}(\Omega)^{d} \times H^{s}(\Omega)$ and that curl $\boldsymbol{u}_{\mid \Omega_{F}}$ belongs to $H^{s+1}\left(\Omega_{F}\right)^{\frac{d(d-1)}{2}}$ for a real number $s, 0<s \leq 1$. The following a priori error estimate holds between the solution $(\boldsymbol{\omega}, \boldsymbol{u}, p)$ of problem (3.1) and the solution $\left(\boldsymbol{\omega}_{h}, \boldsymbol{u}_{h}, p_{h}\right)$ of problem (4.3)

$$
\begin{aligned}
\left\|\boldsymbol{\omega}-\boldsymbol{\omega}_{h}\right\|_{\tilde{H}\left(\operatorname{curl}, \Omega_{F}\right)}+\left\|\boldsymbol{u}-\boldsymbol{u}_{h}\right\|_{H(\operatorname{div}, \Omega)}+\left\|p-p_{h}\right\|_{L^{2}(\Omega)} \\
\quad \leq c\left(h_{\min }^{\Gamma}\right)^{-\frac{1}{2}}\left|\log h_{\min }^{\Gamma}\right| h^{s}\left(\|\boldsymbol{\omega}\|_{H^{s+1}\left(\Omega_{F}\right)} \frac{d(d-1)}{2}+\|\boldsymbol{u}\|_{H^{s}(\Omega)^{d}}+\|p\|_{H^{s}(\Omega)}\right)
\end{aligned}
$$

Remark 4.8. According to ([3], Sect. 4), the quantity $\|\boldsymbol{\omega}\|_{H^{s+1}(\Omega)} \frac{d(d-1)}{2}$ in the previous estimate can be replaced by

$$
\|\boldsymbol{\omega}\|_{H^{s}\left(\Omega_{F}\right)} \frac{d(d-1)}{2}+\|\operatorname{curl} \boldsymbol{\omega}\|_{H^{s}\left(\Omega_{F}\right)^{d}}
$$

there also for any $s, 0<s \leq 1$.

Estimate (4.19) is not optimal but yields the convergence of the discretization under some non restrictive assumptions on the size of the mesh in a neighbourhood of $\Gamma$ and a stronger one on the regularity of the solution. 


\section{A POSTERIORI ANALYSIS OF THE DISCRETIZATION}

We now intend to prove a posteriori error estimates between the solutions $(\boldsymbol{\omega}, \boldsymbol{u}, p)$ of problem (3.1) and $\left(\boldsymbol{\omega}_{h}, \boldsymbol{u}_{h}, p_{h}\right)$ of problem (4.3). We first observe that the error function $U-U_{h}$, with $U=(\boldsymbol{\omega}, \boldsymbol{u})$ and $U_{h}=$ $\left(\boldsymbol{\omega}_{h}, \boldsymbol{u}_{h}\right)$, belongs to $\mathcal{X}$ and satisfies

$$
\begin{aligned}
& \forall \boldsymbol{v} \in W, \quad \mathcal{A}\left(U-U_{h}, \boldsymbol{v}\right)=\langle F, \boldsymbol{v}\rangle, \\
& \forall \boldsymbol{\varphi} \in \tilde{H}_{0}\left(\operatorname{curl}, \Omega_{F}\right), \quad \mathcal{B}\left(U-U_{h}, \boldsymbol{\varphi}\right)=\langle G, \boldsymbol{\varphi}\rangle,
\end{aligned}
$$

where the "residuals" $F$ and $G$ belong to the dual spaces of $H_{0}(\operatorname{div}, \Omega)$ and $\tilde{H}_{0}\left(\mathbf{c u r l}, \Omega_{F}\right)$, respectively, and are defined by

$$
\begin{aligned}
& \forall \boldsymbol{v} \in W, \quad\langle F, \boldsymbol{v}\rangle=\int_{\Omega} \boldsymbol{f} \cdot \boldsymbol{v} \mathrm{d} \boldsymbol{x}-\mu \int_{\Omega_{P}} \boldsymbol{u}_{h} \cdot \boldsymbol{v} \mathrm{d} \boldsymbol{x}-\nu \int_{\Omega_{F}} \operatorname{curl} \boldsymbol{\omega}_{h} \cdot \boldsymbol{v} \mathrm{d} \boldsymbol{x}, \\
& \forall \boldsymbol{\varphi} \in \tilde{H}_{0}\left(\operatorname{curl}, \Omega_{F}\right), \quad\langle G, \boldsymbol{\varphi}\rangle=-\int_{\Omega_{F}} \boldsymbol{\omega}_{h} \cdot \boldsymbol{\varphi} \mathrm{d} \boldsymbol{x}+\int_{\Omega_{F}} \boldsymbol{u}_{h} \cdot \operatorname{curl} \boldsymbol{\varphi} \mathrm{d} \boldsymbol{x} .
\end{aligned}
$$

Deriving a posteriori estimates from these equations requires the following inf-sup condition, which is proven by exactly the same arguments as for Lemma 4.6: there exists a constant $\delta>0$ such that

$$
\forall \varphi \in \tilde{H}_{0}\left(\operatorname{curl}, \Omega_{F}\right), \quad \sup _{V \in \mathcal{X}} \frac{\mathcal{B}(V, \varphi)}{\|V\|_{\mathcal{X}}} \geq \delta\|\varphi\|_{\tilde{H}\left(\operatorname{curl}, \Omega_{F}\right)} .
$$

Indeed, using this condition, we derive the existence of a function $\bar{Z}$ in $\mathcal{X}$ such that

$$
\forall \boldsymbol{\varphi} \in \tilde{H}_{0}\left(\operatorname{curl}, \Omega_{F}\right), \quad \mathcal{B}(\bar{Z}, \varphi)=\langle G, \varphi\rangle
$$

and which satisfies

$$
\|\bar{Z}\|_{\mathcal{X}} \leq \delta^{-1}\|G\|_{\tilde{H}_{0}\left(\operatorname{curl}, \Omega_{F}\right)^{\prime}}
$$

The idea is that, now, the function $U-U_{h}-\bar{Z}$ belongs to $\mathcal{W}$, so that applying (3.8) and a triangle inequality yields the estimate, for a constant $c$ independent of $h$,

$$
\left\|\boldsymbol{\omega}-\boldsymbol{\omega}_{h}\right\|_{\tilde{H}\left(\operatorname{curl}, \Omega_{F}\right)}+\left\|\boldsymbol{u}-\boldsymbol{u}_{h}\right\|_{H(\operatorname{div}, \Omega)} \leq c\left(\|F\|_{W^{\prime}}+\|G\|_{\tilde{H}_{0}\left(\operatorname{curl}, \Omega_{F}\right)^{\prime}}\right) .
$$

So, we must now evaluate the terms in the right-hand side.

We introduce the space

$$
\mathbb{Z}_{h}(\Omega)=\left\{\boldsymbol{g}_{h} \in L^{2}(\Omega)^{d} ; \forall K \in \mathcal{T}_{h}, \boldsymbol{g}_{h \mid K} \in \mathcal{P}_{0}(K)^{d}\right\}
$$

and fix an approximation $\boldsymbol{f}_{h}$ of the data $\boldsymbol{f}$ in $\mathbb{Z}_{h}(\Omega)$. We denote by $\mathcal{E}_{h}$ the set of all edges $(d=2)$ or faces $(d=3)$ of elements of $\mathcal{T}_{h}$ that are not contained in $\partial \Omega$. Next, for each $K$ in $\mathcal{T}_{h}^{F}$, we introduce the set $\mathcal{E}_{K}$ of edges $(d=2)$ or faces $(d=3)$ of $K$ that are not contained in $\Gamma$. Here, $\boldsymbol{n}_{K}$ stands for the unit outward normal vector to $K$ on $\partial K$. For each $K$ in $\mathcal{T}_{h}^{F}$ and for each $e$ in $\mathcal{E}_{K}$, we also denote by $[\cdot]_{e}$ the jump through $e$. Finally the function $\chi^{F}$ is defined on $\mathcal{T}_{h}$ as follows: $\chi^{F}(K)$ is equal to 1 if $K$ belongs to $\mathcal{T}_{h}^{F}$ and to zero otherwise. We also set: $\chi^{P}=1-\chi^{F}$.

We are now in a position to define the two sets of error indicators:

- for each $K$ in $\mathcal{T}_{h}$,

$$
\eta_{K}=\left\|\boldsymbol{f}_{h}-\mu \chi^{P}(K) \boldsymbol{u}_{h}-\nu \chi^{F}(K) \operatorname{curl} \boldsymbol{\omega}_{h}\right\|_{L^{2}(K)^{d}}
$$


- for each $K$ in $\mathcal{T}_{h}^{F}$,

$$
\eta_{K}^{F}=h_{K}\left\|\boldsymbol{\omega}_{h}-\operatorname{curl} \boldsymbol{u}_{h}\right\|_{L^{2}(K)} \frac{d(d-1)}{2}+\sum_{e \in \mathcal{E}_{K}}\left(h_{e}^{\frac{1}{2}}\left\|\left[\boldsymbol{u}_{h} \times \boldsymbol{n}\right]_{e}\right\|_{L^{2}(e)^{d}}+\delta_{d 3} h_{e}^{\frac{3}{2}}\left\|\left[\boldsymbol{\omega}_{h} \cdot \boldsymbol{n}\right]_{e}\right\|_{L^{2}(e)}\right),
$$

where $h_{e}$ denotes the length of $e$ in dimension $d=2$, its diameter in dimension $d=3$, and $\delta$.. stands for the Kronecker symbol (so, $\delta_{d 3}$ is equal to zero in dimension $d=2$, to 1 in dimension $d=3$ ).

Lemma 5.1. The following estimates hold

$$
\|F\|_{W^{\prime}} \leq c\left(\sum_{K \in \mathcal{T}_{h}} \eta_{K}^{2}+\left\|\boldsymbol{f}-\boldsymbol{f}_{h}\right\|_{L^{2}(K)^{d}}^{2}\right)^{\frac{1}{2}}, \quad\|G\|_{\tilde{H}_{0}\left(\operatorname{curl}, \Omega_{F}\right)^{\prime}} \leq c\left(\sum_{K \in \mathcal{T}_{h}^{F}} \lambda_{K}^{F}\left(\eta_{K}^{F}\right)^{2}\right)^{\frac{1}{2}},
$$

where $\lambda_{K}^{F}$ is equal to 1 always in dimension $d=2$ and in dimension $d=3$ only if $\Omega_{F}$ is convex, to $h_{K}^{-\frac{1}{2}}$ in dimension $d=3$ if $\Omega_{F}$ is not convex.

Proof. The first estimate in (5.6) follows from a simple triangle inequality. To obtain the second one, we note that, for any $\varphi_{h}$ in $\mathbb{C}_{h}\left(\Omega_{F}\right)$,

$$
\langle G, \boldsymbol{\varphi}\rangle=-\int_{\Omega_{F}} \boldsymbol{\omega}_{h} \cdot\left(\boldsymbol{\varphi}-\boldsymbol{\varphi}_{h}\right) \mathrm{d} \boldsymbol{x}+\int_{\Omega_{F}} \boldsymbol{u}_{h} \cdot \operatorname{curl}\left(\boldsymbol{\varphi}-\boldsymbol{\varphi}_{h}\right) \mathrm{d} \boldsymbol{x} .
$$

We treat separately the dimensions $d=2$ and $d=3$.

1) In dimension $d=2$, since $\tilde{H}_{0}\left(\mathbf{c u r l}, \Omega_{F}\right)$ coincides with $H_{0}^{1}\left(\Omega_{F}\right)$, we integrate by parts the last term of (5.7) and take $\varphi_{h}$ equal to the image of $\varphi$ by a Clément type regularization operator, which satisfies for all $K$ in $\mathcal{T}_{h}^{F}$ and $e$ in $\mathcal{E}_{K}$,

$$
\left\|\boldsymbol{\varphi}-\boldsymbol{\varphi}_{h}\right\|_{L^{2}(K)} \leq c h_{K}\|\boldsymbol{\varphi}\|_{H^{1}\left(\Delta_{K}\right)} \quad \text { and } \quad\left\|\boldsymbol{\varphi}-\boldsymbol{\varphi}_{h}\right\|_{L^{2}(e)} \leq c h_{e}^{\frac{1}{2}}\|\boldsymbol{\varphi}\|_{H^{1}\left(\Delta_{K}\right)},
$$

where $\Delta_{K}$ stands for the union of the elements of $\mathcal{T}_{h}^{F}$ that intersect $K$. This leads to the desired result.

2) In dimension $d=3$, and for all $\varphi$ in $\tilde{H}_{0}\left(\operatorname{curl}, \Omega_{F}\right)$, we consider the solution $\lambda$ in $H_{0}^{1}\left(\Omega_{F}\right)$ of the problem

$$
\forall \mu \in H_{0}^{1}\left(\Omega_{F}\right), \quad \int_{\Omega_{F}} \operatorname{grad} \lambda \cdot \operatorname{grad} \mu \mathrm{d} \boldsymbol{x}=\int_{\Omega_{F}} \varphi \cdot \operatorname{grad} \mu \mathrm{d} \boldsymbol{x} .
$$

Then, the function $\varphi^{0}=\varphi-\operatorname{grad} \lambda$ belongs to $\tilde{H}_{0}\left(\operatorname{curl}, \Omega_{F}\right)$ and is divergence-free on $\Omega_{F}$. So the pair $\left(\varphi^{0}, \lambda\right)$ belongs ([3], Sect. 2), to $H^{s}(\Omega)^{3} \times H^{s+1}(\Omega)$ with $s=1$ if $\Omega$ is convex, with $s=\frac{1}{2}$ (and even with some $s>\frac{1}{2}$ since $\Omega$ is a polyhedron) otherwise. Next, we observe that, for all $\psi_{h}^{0}$ in $\mathbb{C}_{h}\left(\Omega_{F}\right)$ and $\mu_{h}$ in $H_{0}^{1}\left(\Omega_{F}\right)$ such that its restriction to each $K$ in $\mathcal{T}_{h}^{F}$ belongs to $\mathcal{P}_{1}(K)$,

$$
\langle G, \boldsymbol{\varphi}\rangle=-\int_{\Omega_{F}} \boldsymbol{\omega}_{h} \cdot\left(\boldsymbol{\varphi}^{0}-\boldsymbol{\psi}_{h}^{0}\right) \mathrm{d} \boldsymbol{x}-\int_{\Omega_{F}} \boldsymbol{\omega}_{h} \cdot \operatorname{grad}\left(\lambda-\mu_{h}\right) \mathrm{d} \boldsymbol{x}+\int_{\Omega_{F}} \boldsymbol{u}_{h} \cdot \operatorname{curl}\left(\boldsymbol{\varphi}^{0}-\boldsymbol{\psi}_{h}^{0}\right) \mathrm{d} \boldsymbol{x} .
$$

By integrating by parts on each $K$ and noting that $\operatorname{div} \boldsymbol{\omega}_{h}$ is zero on each $K$, we obtain

$$
\begin{aligned}
\langle G, \boldsymbol{\varphi}\rangle=\sum_{K \in \mathcal{T}_{h}^{F}}\left(-\int_{K}\left(\boldsymbol{\omega}_{h}-\operatorname{curl} \boldsymbol{u}_{h}\right) \cdot\left(\boldsymbol{\varphi}^{0}-\boldsymbol{\psi}_{h}^{0}\right) \mathrm{d} \boldsymbol{x}\right. & \\
& \left.+\frac{1}{2} \sum_{e \in \mathcal{E}_{K}}\left(\int_{e}\left[\boldsymbol{u}_{h} \times \boldsymbol{n}\right] \cdot\left(\boldsymbol{\varphi}^{0}-\boldsymbol{\psi}_{h}^{0}\right) \mathrm{d} \boldsymbol{\tau}+\int_{e}\left[\boldsymbol{\omega}_{h} \cdot \boldsymbol{n}\right]\left(\lambda-\mu_{h}\right) \mathrm{d} \boldsymbol{\tau}\right)\right) .
\end{aligned}
$$


So we take $\boldsymbol{\psi}_{h}^{0}$ equal to the image of $\boldsymbol{\varphi}^{0}$ by the Raviart-Thomas operator (see below for its exact definition) and $\mu_{h}$ equal to the image of $\lambda$ by the Clément type regularization operator quoted above. By using the regularity properties of $\varphi^{0}$ and $\lambda$, we derive the desired estimate.

Combining estimate (5.3) with Lemma 5.1, we obtain the first a posteriori estimate.

Corollary 5.2. The following a posteriori error estimate holds between the solution $(\boldsymbol{\omega}, \boldsymbol{u}, p)$ of problem (3.1) and the solution $\left(\boldsymbol{\omega}_{h}, \boldsymbol{u}_{h}, p_{h}\right)$ of problem (4.3)

$$
\left\|\boldsymbol{\omega}-\boldsymbol{\omega}_{h}\right\|_{\tilde{H}\left(\mathbf{c u r l}, \Omega_{F}\right)}+\left\|\boldsymbol{u}-\boldsymbol{u}_{h}\right\|_{H(\mathrm{div}, \Omega)} \leq c\left(\sum_{K \in \mathcal{T}_{h}}\left(\eta_{K}^{2}+\left\|\boldsymbol{f}-\boldsymbol{f}_{h}\right\|_{L^{2}(K)^{d}}^{2}\right)+\sum_{K \in \mathcal{T}_{h}^{F}} \lambda_{K}^{F}\left(\eta_{K}^{F}\right)^{2}\right)^{\frac{1}{2}} .
$$

A further argument is needed to obtain an error estimate on the pressure. Indeed this relies on the residual equation

$$
\forall \boldsymbol{v} \in H(\operatorname{div}, \Omega), \quad \mathcal{A}\left(U-U_{h}, \boldsymbol{v}\right)+b\left(\boldsymbol{v}, p-p_{h}\right)=\langle F, \boldsymbol{v}\rangle-b\left(\boldsymbol{v}, p_{h}\right) .
$$

Moreover, it can be noted that, for all $\boldsymbol{v}_{h}$ in $\mathbb{D}_{h}(\Omega)$,

$$
\langle F, \boldsymbol{v}\rangle-b\left(\boldsymbol{v}, p_{h}\right)=\left\langle F, \boldsymbol{v}-\boldsymbol{v}_{h}\right\rangle-b\left(\boldsymbol{v}-\boldsymbol{v}_{h}, p_{h}\right) .
$$

In a further step, we introduce the Raviart-Thomas operator: for any smooth enough vectorial function $\boldsymbol{v}, R_{h} \boldsymbol{v}$ belongs to $\mathbb{D}_{h}(\Omega)$ and satisfies

$$
\forall e \in \mathcal{E}_{h}, \quad \int_{e}\left(\boldsymbol{v}-R_{h} \boldsymbol{v}\right) \cdot \boldsymbol{n} \mathrm{d} \tau=0
$$

Moreover, this operator satisfies, see ([26], Thm. 3) or ([24], Thm. 4), for all functions $\boldsymbol{v}$ in $H^{1}(\Omega)^{d}$ and for all $K$ in $\mathcal{T}_{h}$,

$$
\left\|\boldsymbol{v}-R_{h} \boldsymbol{v}\right\|_{L^{2}(K)^{d}} \leq c h_{K}\|\boldsymbol{v}\|_{H^{1}(K)^{d}} .
$$

The idea here is that, thanks to the definition of $\mathbb{M}_{h}(\Omega)$,

$$
b\left(\boldsymbol{v}-R_{h} \boldsymbol{v}, p_{h}\right)=0 .
$$

Proposition 5.3. The following a posteriori error estimate holds between the solution $(\boldsymbol{\omega}, \boldsymbol{u}, p)$ of problem (3.1) and the solution $\left(\boldsymbol{\omega}_{h}, \boldsymbol{u}_{h}, p_{h}\right)$ of problem (4.3)

$$
\left\|p-p_{h}\right\|_{L^{2}(\Omega)} \leq c\left(\sum_{K \in \mathcal{T}_{h}} h_{K}^{2}\left(\eta_{K}^{2}+\left\|\boldsymbol{f}-\boldsymbol{f}_{h}\right\|_{L^{2}(K)^{d}}^{2}\right)\right)^{\frac{1}{2}}+\left\|\boldsymbol{\omega}-\boldsymbol{\omega}_{h}\right\|_{\tilde{H}\left(\operatorname{curl}, \Omega_{F}\right)}+\left\|\boldsymbol{u}-\boldsymbol{u}_{h}\right\|_{H(\operatorname{div}, \Omega)} .
$$

Proof. The proof relies on the standard inf-sup condition ([18], Chap. I, Cor. 2.4): for a positive constant $\tilde{\beta}$,

$$
\forall q \in L_{0}^{2}(\Omega), \quad \sup _{\boldsymbol{v} \in H_{0}^{1}(\Omega)^{d}} \frac{b(\boldsymbol{v}, q)}{\|\boldsymbol{v}\|_{H^{1}(\Omega)^{d}}} \geq \tilde{\beta}\|q\|_{L^{2}(\Omega)} .
$$

By using this inf-sup condition and combining equation (5.9) with (5.10) and (5.13), we obtain

$$
\left\|p-p_{h}\right\|_{L^{2}(\Omega)} \leq \tilde{\beta}^{-1} \sup _{\boldsymbol{v} \in H_{0}^{1}(\Omega)^{d}} \frac{\left\langle F, \boldsymbol{v}-R_{h} \boldsymbol{v}\right\rangle-\mathcal{A}\left(U-U_{h}, \boldsymbol{v}\right)}{\|\boldsymbol{v}\|_{H^{1}(\Omega)}} .
$$

As for Lemma 5.1, we have

$$
\left\langle F, \boldsymbol{v}-R_{h} \boldsymbol{v}\right\rangle \leq \sum_{K \in \mathcal{T}_{h}}\left\|\boldsymbol{f}-\mu \chi^{P}(K) \boldsymbol{u}_{h}-\nu \chi^{F}(K) \operatorname{curl} \boldsymbol{\omega}_{h}\right\|_{L^{2}(K)^{d}}\left\|\boldsymbol{v}-R_{h} \boldsymbol{v}\right\|_{L^{2}(K)^{d}}
$$


So using (5.12) yields

$$
\left\langle F, \boldsymbol{v}-R_{h} \boldsymbol{v}\right\rangle \leq c\left(\sum_{K \in \mathcal{T}_{h}} h_{K}^{2}\left(\left\|\boldsymbol{f}_{h}-\mu \chi^{P}(K) \boldsymbol{u}_{h}-\nu \chi^{F}(K) \operatorname{curl} \boldsymbol{\omega}_{h}\right\|_{L^{2}(K)^{d}}^{2}+\left\|\boldsymbol{f}-\boldsymbol{f}_{h}\right\|_{L^{2}(K)^{d}}\right)\right)^{\frac{1}{2}}\|\boldsymbol{v}\|_{H^{1}(\Omega)^{d}} .
$$

On the other hand, it follows from the continuity of the form $\mathcal{A}(\cdot, \cdot)$ that

$$
\mathcal{A}\left(U-U_{h}, \boldsymbol{v}\right) \leq\left(\left\|\boldsymbol{\omega}-\boldsymbol{\omega}_{h}\right\|_{\tilde{H}\left(\operatorname{curl}, \Omega_{F}\right)}+\left\|\boldsymbol{u}-\boldsymbol{u}_{h}\right\|_{H(\operatorname{div}, \Omega)}\right)\|\boldsymbol{v}\|_{H(\operatorname{div}, \Omega)} .
$$

Combining all this yields the desired estimate.

We now prove the upper bounds for the two types of indicators.

Proposition 5.4. The following estimate holds for each indicator $\eta_{K}, K \in \mathcal{T}_{h}$, defined in (5.4)

$$
\eta_{K} \leq c\left(\chi_{F}(K)\left\|\boldsymbol{\omega}-\boldsymbol{\omega}_{h}\right\|_{\tilde{H}(\operatorname{curl}, K)}+\chi_{P}(K)\left\|\boldsymbol{u}-\boldsymbol{u}_{h}\right\|_{H(\mathrm{div}, K)}+h_{K}^{-1}\left\|p-p_{h}\right\|_{L^{2}(K)}+\left\|\boldsymbol{f}-\boldsymbol{f}_{h}\right\|_{L^{2}(K)^{d}}\right) .
$$

Proof. In equation (5.9), we take $\boldsymbol{v}$ equal to

$$
\boldsymbol{v}= \begin{cases}\left(\boldsymbol{f}_{h}-\mu \chi^{P}(K) \boldsymbol{u}_{h}-\nu \chi^{F}(K) \operatorname{curl} \boldsymbol{\omega}_{h}\right) \psi_{K} & \text { on } K, \\ \mathbf{0} & \text { elsewhere }\end{cases}
$$

where $\psi_{K}$ denotes the bubble function on $K$ equal to the product of the $d+1$ barycentric coordinates associated with the vertices of $K$. This gives

$$
\left\|\left(\boldsymbol{f}_{h}-\mu \chi^{P}(K) \boldsymbol{u}_{h}-\nu \chi^{F}(K) \operatorname{curl} \boldsymbol{\omega}_{h}\right) \psi_{K}^{\frac{1}{2}}\right\|_{L^{2}(K)^{d}}^{2} \leq \mathcal{A}\left(U-U_{h}, \boldsymbol{v}\right)+b(\boldsymbol{v}, p)-\int_{K}\left(\boldsymbol{f}-\boldsymbol{f}_{h}\right) \cdot \boldsymbol{v} \mathrm{d} \boldsymbol{x} .
$$

We have

$$
\mathcal{A}\left(U-U_{h}, \boldsymbol{v}\right) \leq\left(\chi_{F}(K)\left\|\boldsymbol{\omega}-\boldsymbol{\omega}_{h}\right\|_{\tilde{H}(\mathbf{c u r l}, K)}+\chi_{P}(K)\left\|\boldsymbol{u}-\boldsymbol{u}_{h}\right\|_{H(\mathrm{div}, K)}\right)\|\boldsymbol{v}\|_{L^{2}(K)^{d}},
$$

and

$$
\int_{K}\left(\boldsymbol{f}-\boldsymbol{f}_{h}\right) \cdot \boldsymbol{v} \mathrm{d} \boldsymbol{x} \leq\left\|\boldsymbol{f}-\boldsymbol{f}_{h}\right\|_{L^{2}(K)^{d}}\|\boldsymbol{v}\|_{L^{2}(K)^{d}} .
$$

On the other hand, we write

$$
b(\boldsymbol{v}, p)=b\left(\boldsymbol{v}, p-p_{h}\right)+b\left(\boldsymbol{v}, p_{h}\right) .
$$

Since $\boldsymbol{v}$ is polynomial with degree $\leq d+1$, we use an inverse inequality to derive

$$
b\left(\boldsymbol{v}, p-p_{h}\right) \leq c h_{K}^{-1}\left\|p-p_{h}\right\|_{L^{2}(K)}\|\boldsymbol{v}\|_{L^{2}(K)^{d}} .
$$

We also observe that, since both $\boldsymbol{f}_{h}-\mu \chi^{P}(K) \boldsymbol{u}_{h}-\nu \chi^{F}(K) \operatorname{curl} \boldsymbol{\omega}_{h}$ and $p_{h}$ are constant on $K$,

$$
b\left(\boldsymbol{v}, p_{h}\right)=-\left(\boldsymbol{f}_{h}-\mu \chi^{P}(K) \boldsymbol{u}_{h}-\nu \chi^{F}(K) \operatorname{curl} \boldsymbol{\omega}_{h}\right)_{\mid K} p_{h \mid K} \int_{K} \operatorname{div} \psi_{K} \mathrm{~d} \boldsymbol{x}=0 .
$$

Combining all this and noting that, since $\psi_{K}$ takes its values in $[0,1]$,

$$
\|\boldsymbol{v}\|_{L^{2}(K)^{d}} \leq\left\|\left(\boldsymbol{f}_{h}-\mu \chi^{P}(K) \boldsymbol{u}_{h}-\nu \chi^{F}(K) \operatorname{curl} \boldsymbol{\omega}_{h}\right) \psi_{K}^{\frac{1}{2}}\right\|_{L^{2}(K)^{d}},
$$


we derive

$$
\begin{aligned}
\left\|\left(\boldsymbol{f}_{h}-\mu \chi^{P}(K) \boldsymbol{u}_{h}-\nu \chi^{F}(K) \operatorname{curl} \boldsymbol{\omega}_{h}\right) \psi_{K}^{\frac{1}{2}}\right\|_{L^{2}(K)^{d}} \leq c\left(\chi_{F}(K)\left\|\boldsymbol{\omega}-\boldsymbol{\omega}_{h}\right\|_{\tilde{H}(\operatorname{curl}, K)}\right. & \\
& \left.+\chi_{P}(K)\left\|\boldsymbol{u}-\boldsymbol{u}_{h}\right\|_{H(\operatorname{div}, K)}+h_{K}^{-1}\left\|p-p_{h}\right\|_{L^{2}(K)}+\left\|\boldsymbol{f}-\boldsymbol{f}_{h}\right\|_{L^{2}(K)^{d}}\right) .
\end{aligned}
$$

Finally, by combining this line with the inverse inequality

$$
\forall w \in \mathcal{P}_{0}(K), \quad\|w\|_{L^{2}(K)} \leq c\left\|w \psi_{K}^{\frac{1}{2}}\right\|_{L^{2}(K)},
$$

we obtain the desired estimate.

Proposition 5.5. The following estimate holds for each indicator $\eta_{K}^{F}, K \in \mathcal{T}_{h}^{F}$, defined in (5.5)

$$
\eta_{K}^{F} \leq c\left(\left\|\boldsymbol{\omega}-\boldsymbol{\omega}_{h}\right\|_{\tilde{H}\left(\operatorname{curl}, \Delta_{K}\right)}+\left\|\boldsymbol{u}-\boldsymbol{u}_{h}\right\|_{H\left(\operatorname{div}, \Delta_{K}\right)}\right)
$$

where $\Delta_{K}$ denotes the union of elements of $\mathcal{T}_{h}^{F}$ that share at least an edge $(d=2)$ or a face $(d=3)$ with $K$.

Proof. This estimate relies on the second line of (5.1). Indeed, we note that, for all $\varphi$ in $\tilde{H}_{0}\left(\operatorname{curl}, \Omega_{F}\right)$,

$$
\langle G, \boldsymbol{\varphi}\rangle=\sum_{K \in \mathcal{T}_{h}^{F}}\left(-\int_{K}\left(\boldsymbol{\omega}_{h}-\operatorname{curl} \boldsymbol{u}_{h}\right) \cdot \boldsymbol{\varphi} \mathrm{d} \boldsymbol{x}+\frac{1}{2} \sum_{e \in \mathcal{E}_{K}} \int_{e}\left[\boldsymbol{u}_{h} \times \boldsymbol{n}\right]_{e} \cdot \boldsymbol{\varphi} \mathrm{d} \boldsymbol{\tau}\right)
$$

1) First, we take $\varphi$ equal to

$$
\boldsymbol{\varphi}= \begin{cases}\left(\boldsymbol{\omega}_{h}-\operatorname{curl} \boldsymbol{u}_{h}\right) \psi_{K} & \text { on } K, \\ \mathbf{0} & \text { elsewhere. }\end{cases}
$$

Exactly the same arguments as in the proof of Proposition 5.4 lead to the estimate

$$
\left\|\boldsymbol{\omega}_{h}-\operatorname{curl} \boldsymbol{u}_{h}\right\|_{L^{2}(K)^{\frac{d(d-1)}{2}}} \leq c\left(\left\|\boldsymbol{\omega}-\boldsymbol{\omega}_{h}\right\|_{L^{2}(K)^{\frac{d(d-1)}{2}}}+h_{K}^{-1}\left\|\boldsymbol{u}-\boldsymbol{u}_{h}\right\|_{H(\operatorname{div}, K)}\right) .
$$

2) Second, for any edge $e$ in $\mathcal{E}_{K}$, let $K^{\prime}$ denote the other element of $\mathcal{T}_{h}^{F}$ which contains $e$. Let $\mathcal{L}_{e}$ be a lifting operator from polynomials defined on $e$ into polynomials defined on $K$ and $K^{\prime}$, which is constructed by affine transformations from a fixed operator on the reference element. We now take $\varphi$ equal to

$$
\boldsymbol{\varphi}= \begin{cases}\mathcal{L}_{e}\left(\left[\boldsymbol{u}_{h} \times \boldsymbol{n}\right]_{e}\right) \psi_{e} & \text { on } K \cup K^{\prime} \\ \mathbf{0} & \text { elsewhere }\end{cases}
$$

where $\psi_{e}$ denotes the bubble function on $e$, equal to the product of the $d$ barycentric coordinates associated with the vertices of $e$. There also standard arguments (see [29], Sect. 1.2) yield

$$
\left\|\left[\boldsymbol{u}_{h} \times \boldsymbol{n}\right]_{e}\right\|_{L^{2}(e)^{d}} \leq c\left(\left\|\boldsymbol{\omega}-\boldsymbol{\omega}_{h}\right\|_{L^{2}\left(K \cup K^{\prime}\right)} \frac{d(d-1)}{2}+h_{e}^{-\frac{1}{2}}\left\|\boldsymbol{u}-\boldsymbol{u}_{h}\right\|_{H\left(\operatorname{div}, K \cup K^{\prime}\right)}\right.
$$

3) Finally, in dimension $d=3$, taking $\varphi$ equal to $\operatorname{grad} \mu$ in the second line of (5.1) for any function $\mu$ of $H_{0}^{1}\left(\Omega_{F}\right)$ leads to the equation

$$
\int_{\Omega_{F}}\left(\boldsymbol{\omega}-\boldsymbol{\omega}_{h}\right) \cdot \operatorname{grad} \mu \mathrm{d} \boldsymbol{x}=\frac{1}{2} \sum_{K \in \mathcal{T}_{h}^{F}} \sum_{e \in \mathcal{E}_{K}} \int_{e}\left[\boldsymbol{\omega}_{h} \cdot \boldsymbol{n}\right]_{e} \mu \mathrm{d} \tau
$$

There also and with the same notation as previously, taking

$$
\mu= \begin{cases}\mathcal{L}_{e}\left(\left[\boldsymbol{\omega}_{h} \cdot \boldsymbol{n}\right]_{e}\right) \psi_{e} & \text { on } K \cup K^{\prime} \\ 0 & \text { elsewhere }\end{cases}
$$


gives the estimate

$$
\left\|\left[\boldsymbol{\omega}_{h} \cdot \boldsymbol{n}\right]_{e}\right\|_{L^{2}(e)} \leq c h_{e}^{-\frac{1}{2}}\left\|\boldsymbol{\omega}-\boldsymbol{\omega}_{h}\right\|_{L^{2}\left(K \cup K^{\prime}\right)} \frac{d(d-1)}{2} .
$$

The desired estimate follows from (5.19), (5.20) and (5.21).

Estimates (5.8), (5.14), (5.16) and (5.17) are not fully optimal. Indeed, the global error

$$
\left\|\boldsymbol{\omega}-\boldsymbol{\omega}_{h}\right\|_{\tilde{H}(\mathbf{c u r l}, K)}+\left\|\boldsymbol{u}-\boldsymbol{u}_{h}\right\|_{H(\operatorname{div}, K)}+\left\|p-p_{h}\right\|_{L^{2}(K)},
$$

is up to the terms involving the data bounded by a constant independent of $h$ times the Hilbertian sum of the indicators

$$
\left(\sum_{K \in \mathcal{T}_{h}} \eta_{K}^{2}+\sum_{K \in \mathcal{T}_{h}^{F}} \lambda_{K}^{F}\left(\eta_{K}^{F}\right)^{2}\right)^{\frac{1}{2}},
$$

where $\lambda_{h}^{F}$ is equal to 1 either in dimension $d=2$ or when $\Omega_{F}$ is convex in dimension $d=3$. However this last quantity is only bounded by the error times a constant behaving like $\left(\min _{K \in \mathcal{T}_{h}} h_{K}\right)^{-1}$. Nevertheless,

1) the lack of optimality when the $\lambda_{K}^{F}$ are equal to 1 only concerns the error on the pressure (when this error is zero, the estimates are optimal);

2) exactly the same lack of optimality has been observed in ([8], Sect. 4), in the simpler case of uncoupled Darcy equations discretized by the Raviart-Thomas element.

\section{SOME NUMERICAL EXPERIMENTS}

The numerical tests have been performed with the code freefem ++ , see [20], in the two-dimensional case. We work with a different boundary condition on $\partial \Omega$, namely a given pressure, and, in order to do that, we introduce the modified space

$$
\overline{\mathbb{D}}_{h}(\Omega)=\left\{\boldsymbol{v}_{h} \in H(\operatorname{div}, \Omega) ; \forall K \in \mathcal{T}_{h}, \boldsymbol{v}_{h \mid K} \in P_{K}\right\},
$$

which contains $\mathbb{D}_{h}(\Omega)$ but where the nullity of normal traces on $\partial \Omega$ is no longer enforced. For some parameter $\varepsilon$, we solve the modified problem (with $\mu=\nu=1$ for simplicity)

Find $\left(\boldsymbol{\omega}_{h}, \boldsymbol{u}_{h}, p_{h}\right)$ in $\mathbb{C}_{h}\left(\Omega_{F}\right) \times \overline{\mathbb{D}}_{h}(\Omega) \times \mathbb{M}_{h}(\Omega)$ such that

$$
\begin{aligned}
& \forall \boldsymbol{\varphi}_{h} \in \mathbb{C}_{h}\left(\Omega_{F}\right), \quad-\varepsilon \int_{\Omega_{F}} \boldsymbol{\omega}_{h} \cdot \boldsymbol{\varphi}_{h} \mathrm{~d} \boldsymbol{x}+\int_{\Omega_{F}} \boldsymbol{\omega}_{h} \cdot \boldsymbol{\varphi}_{h} \mathrm{~d} \boldsymbol{x}-\int_{\Omega_{F}} \boldsymbol{u}_{h} \cdot \operatorname{curl} \boldsymbol{\varphi}_{h} \mathrm{~d} \boldsymbol{x}=0, \\
& \forall \boldsymbol{v}_{h} \in \overline{\mathbb{D}}_{h}(\Omega), \quad \varepsilon \int_{\Omega} \boldsymbol{u}_{h}(\boldsymbol{x}) \cdot \boldsymbol{v}_{h}(\boldsymbol{x}) \mathrm{d} \boldsymbol{x}+\int_{\Omega_{P}} \boldsymbol{u}_{h} \cdot \boldsymbol{v}_{h} \mathrm{~d} \boldsymbol{x}+\int_{\Omega_{F}} \boldsymbol{c u r l} \boldsymbol{\omega}_{h} \cdot \boldsymbol{v}_{h} \mathrm{~d} \boldsymbol{x}+b\left(\boldsymbol{v}_{h}, p_{h}\right) \\
& =\int_{\partial \Omega}(x+y)\left(v_{h x} n_{x}+v_{h y} n_{y}\right) \mathrm{d} \tau, \\
& \forall q_{h} \in \mathbb{M}_{h}(\Omega), \quad-\varepsilon \int_{\Omega} p_{h}(\boldsymbol{x}) q_{h}(\boldsymbol{x}) \mathrm{d} \boldsymbol{x}+b\left(\boldsymbol{u}_{h}, q_{h}\right)=0,
\end{aligned}
$$

where $v_{h x}$ and $v_{h y}$, respectively $n_{x}$ and $n_{y}$, stand for the components of $\boldsymbol{v}_{h}$, respectively of $\boldsymbol{n}$. Indeed, in comparison with problem (4.3), some regularization terms have been added because the linear system is solved by a Gauss block-factorization method and the blocks (one block per mesh node) are not invertible otherwise. We have checked that there is no dependency of the solution upon $\varepsilon$, which is fixed here equal to $10^{-10}$.

\section{VALIDATION TESTS}

To validate the numerics we present three tests, according as if $\Omega_{F}$ is empty, is the whole domain $\Omega$ or is a non empty part of $\Omega$. In all of them, $\Omega$ is a rectangle, we take $\mu=\nu=1$ for simplicity and we work with a uniform mesh. 


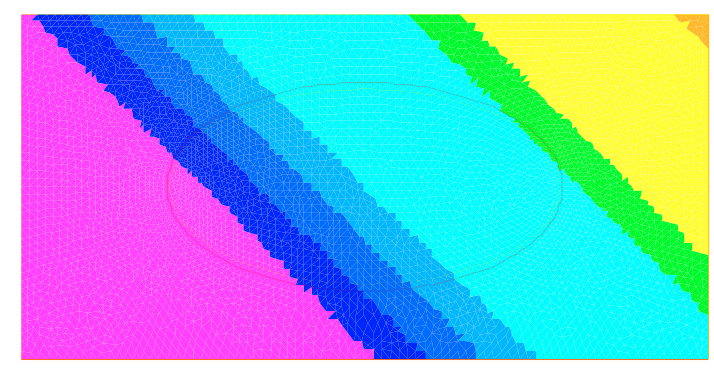

Figure 2. The pressure in Case 1.
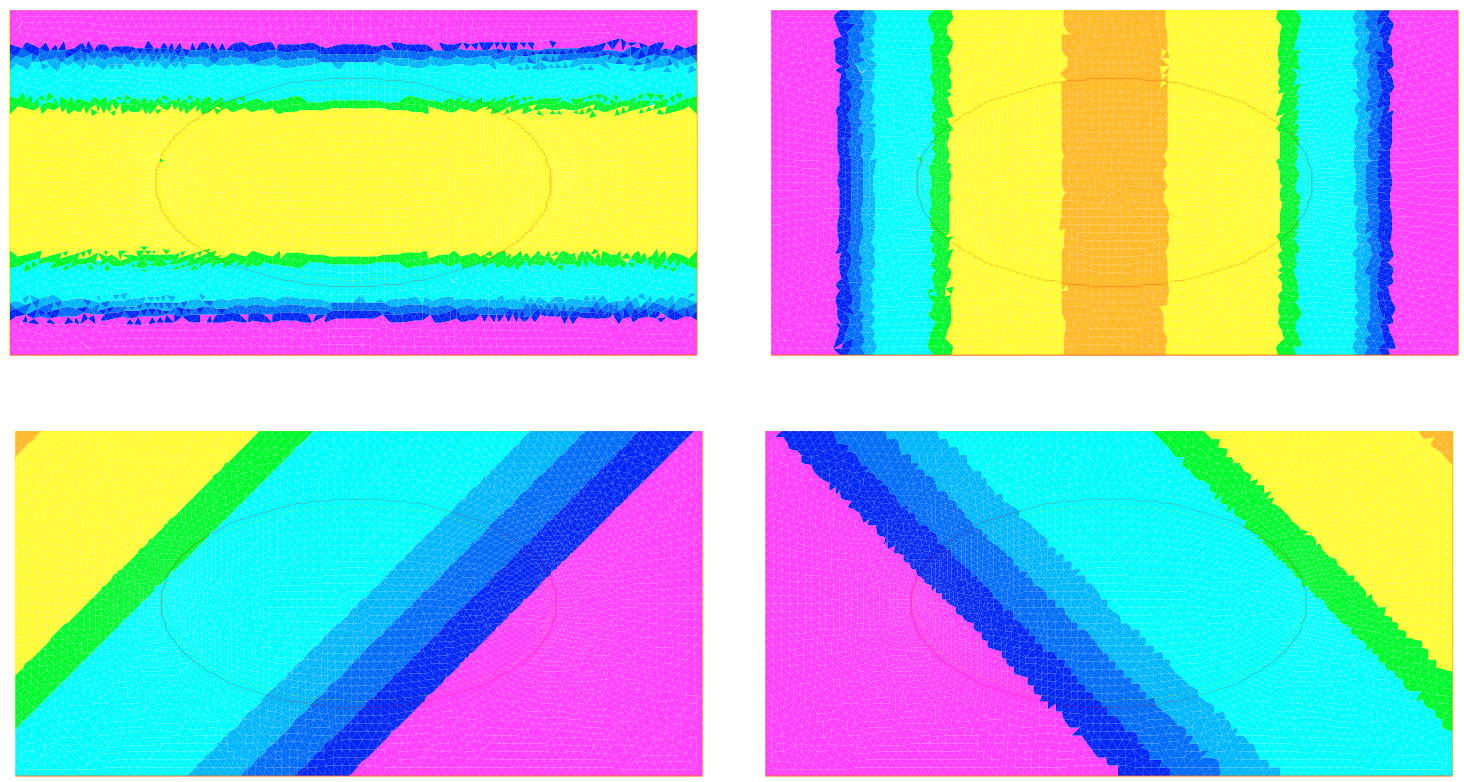

Figure 3. The velocity, vorticity and pressure in Case 2.

Case 1. Darcy equations everywhere $\left(\Omega_{F}=\emptyset, \Omega_{P}=\Omega\right)$.

The analytical solution is given by

$$
\boldsymbol{u}(x, y)=(1,1), \quad p(x, y)=-x-y
$$

In Figure 2, the iso-level curves of the Darcy (hydrostatic) pressure are presented. So the analytical pressure is well recaptured (the velocity is not drawn since it coincides with the constant analytical velocity everywhere).

Case 2. Stokes equations everywhere $\left(\Omega_{F}=\Omega, \Omega_{P}=\emptyset\right)$.

The analytical solution is given by

$$
\boldsymbol{\omega}(x, y)=y-x, \quad \boldsymbol{u}(x, y)=\left(-\frac{1}{2} y^{2},-\frac{1}{2} x^{2}\right), \quad p(x, y)=-x-y
$$

Figure 3 presents, from left to right and from top to bottom, the horizontal velocity, the vertical velocity, the vorticity, the pressure. There also, the analytical solution is recaptured correctly. 

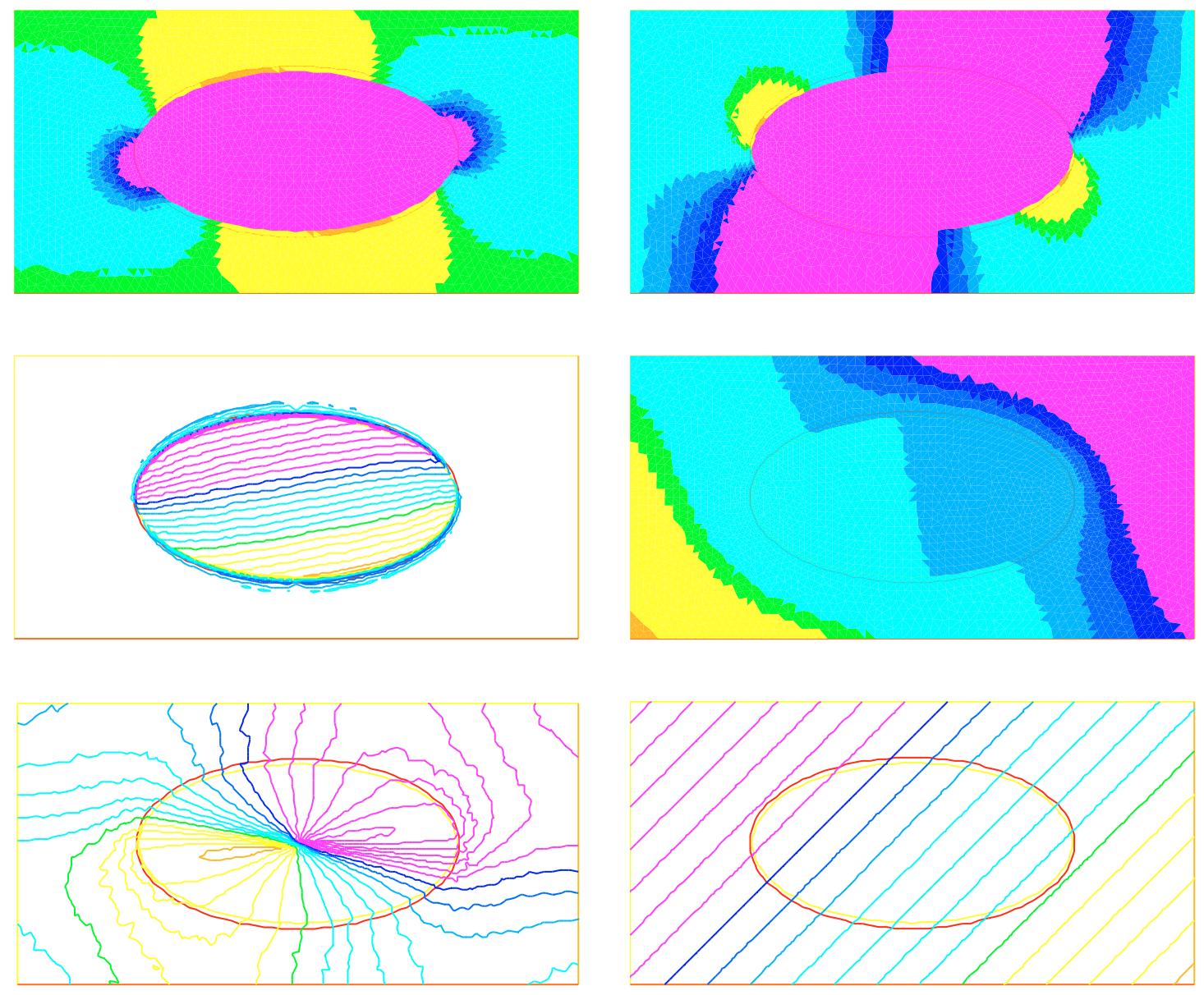

FiguRE 4. The velocity, vorticity, pressure and others in Case 3.

Case 3. Coupling Darcy and Stokes equations.

Here, $\Omega_{F}$ is the interior of an ellipse. In Figure 4, are presented, from left to right and from top to bottom, the horizontal velocity, the vertical velocity, the vorticity and the pressure. The last two plots show, on the left, the continuity of $\boldsymbol{u} \cdot \boldsymbol{n}$ (extended in the whole domain) and, on the right, the streamlines of the flow.

We observe that we recover the analytical solution for the first two cases. We see also in Figure 4 that the tangential component of the velocity and the normal component of the vorticity are indeed discontinuous.

\section{Mesh ADAPTATION}

We still consider Case 3 and perform mesh adaptivity relying on the error indicators defined in (5.4) and (5.5). The adaptivity strategy is the same as described in ([17], Sect. 20.9), for instance, and relies on the generation of new, adapted, meshes proposed in ([16], Chap. 21). Figure 5 presents, from left to right and from top to bottom, the adapted mesh, the horizontal velocity, the vertical velocity, the vorticity, the pressure and the streamlines of the flow.

\section{SEEPAGE}

To illustrate seeping fluids, we consider a cavity problem with a porous bottom layer: the domain $\Omega$ is still a rectangle, the domain $\Omega_{P}$ is a quadrilateral on the bottom of the rectangle. The viscosity $\nu$ is still equal to 1 and we consider two cases, one with porosity coefficient $\mu^{-1}$ equal to 100 in Figure 6 and another one 

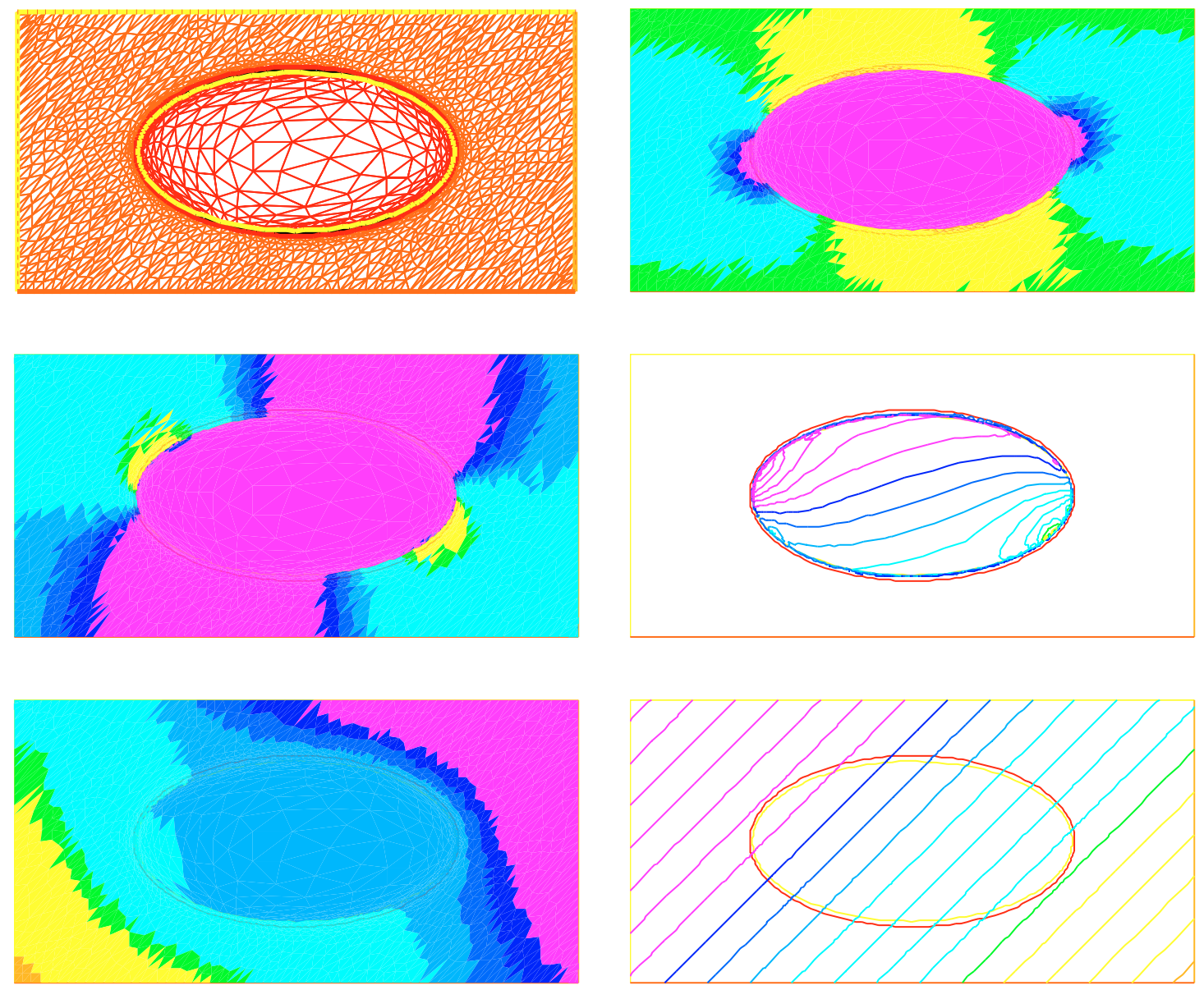

FiguRE 5. The adapted mesh and others in Case 3.

with $\mu^{-1}$ equal to 0.01 in Figure 7. In these figures are presented, from left to right and from top to bottom, the horizontal velocity, the vertical velocity, the vorticity, the pressure and the streamlines of the flow.

\section{APPENDIX}

Let $\overline{\mathbb{H}}_{h}\left(\Omega_{F}\right)$ be the space of functions in $L^{2}\left(\Omega_{F}\right)$ such that their restriction to each $K$ in $\mathcal{T}_{h}^{F}$ belongs $\mathcal{P}_{1}(K)$. It can be noted that the traces of such functions do not belong to $H^{\frac{1}{2}}(\Gamma)$ but only to $H^{\frac{1}{2}-\varepsilon}(\Gamma)$ for all positive $\varepsilon$. The aim of this appendix is to prove the following estimate: for all $\varepsilon, 0<\varepsilon<\frac{1}{2}$, there exists a constant $\alpha(\varepsilon)$ such that

$$
\forall \mu_{h} \in \tilde{\mathbb{H}}_{h}\left(\Omega_{F}\right), \quad \int_{\Gamma} \boldsymbol{v}_{h} \cdot \boldsymbol{n} \mu_{h} \mathrm{~d} \boldsymbol{\tau} \leq \alpha(\varepsilon)\left(h_{\min }^{\Gamma}\right)^{-\frac{1}{2}-\varepsilon}\left\|\boldsymbol{v}_{h}\right\|_{L^{2}\left(\Omega_{P}\right)^{d}}\left\|\mu_{h}\right\|_{H_{h}^{1}\left(\Omega_{F}\right)},
$$

where $\|\cdot\|_{H_{h}^{1}\left(\Omega_{F}\right)}$ denotes the $H^{1}\left(\Omega_{F}\right)$ broken norm associated with the mesh $\mathcal{T}_{h}^{F}$.

Let $\mathcal{E}_{h}^{\Gamma}$ denote the set of edges $(d=2)$ or faces $(d=3)$ of $\mathcal{T}_{h}^{F}$ that are contained in $\Gamma$. For each $e$ in $\mathcal{E}_{h}^{\Gamma}$, we denote by $K_{e}^{F}$, respectively $K_{e}^{P}$, the element of $\mathcal{T}_{h}^{F}$, respectively of $\mathcal{T}_{h} \backslash \mathcal{T}_{h}^{F}$, that contains $e$. We have

$$
\int_{\Gamma} \boldsymbol{v}_{h} \cdot \boldsymbol{n} \mu_{h} \mathrm{~d} \boldsymbol{\tau}=\sum_{e \in \mathcal{E}_{h}^{\Gamma}} \int_{e} \boldsymbol{v}_{h} \cdot \boldsymbol{n} \mu_{h} \mathrm{~d} \boldsymbol{\tau}
$$



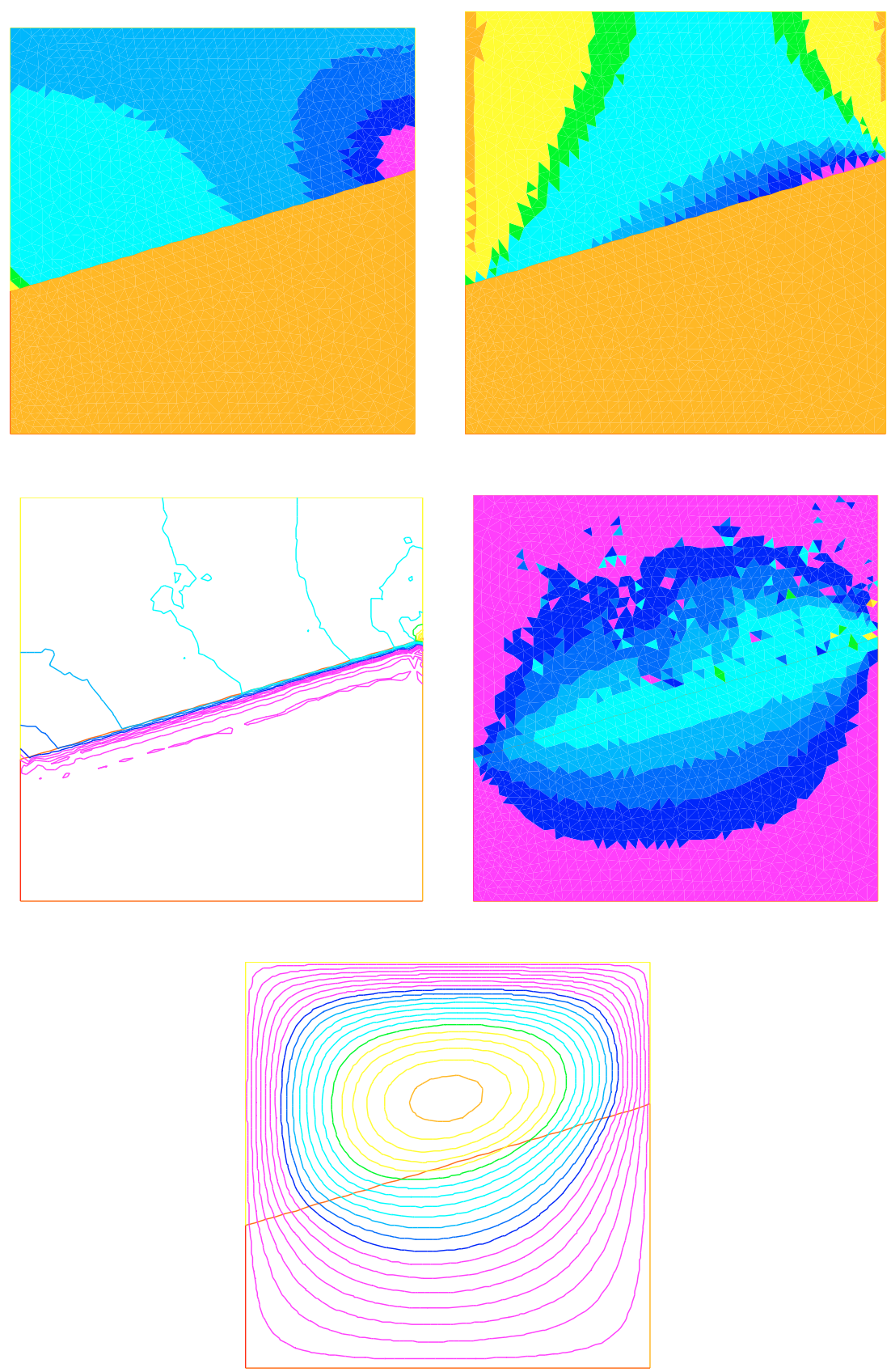

Figure 6. A seeping fluid (porosity 100).

whence

$$
\int_{\Gamma} \boldsymbol{v}_{h} \cdot \boldsymbol{n} \mu_{h} \mathrm{~d} \boldsymbol{\tau} \leq\left(\sum_{e \in \mathcal{E}_{h}^{\Gamma}}\left\|\boldsymbol{v}_{h} \cdot \boldsymbol{n}\right\|_{H^{\varepsilon-\frac{1}{2}}(e)}^{2}\right)^{\frac{1}{2}}\left(\sum_{e \in \mathcal{E}_{h}^{\Gamma}}\left\|\mu_{h}\right\|_{H^{\frac{1}{2}-\varepsilon}(e)}^{2}\right)^{\frac{1}{2}} .
$$

We now bound each of the two terms in the right-hand side. 

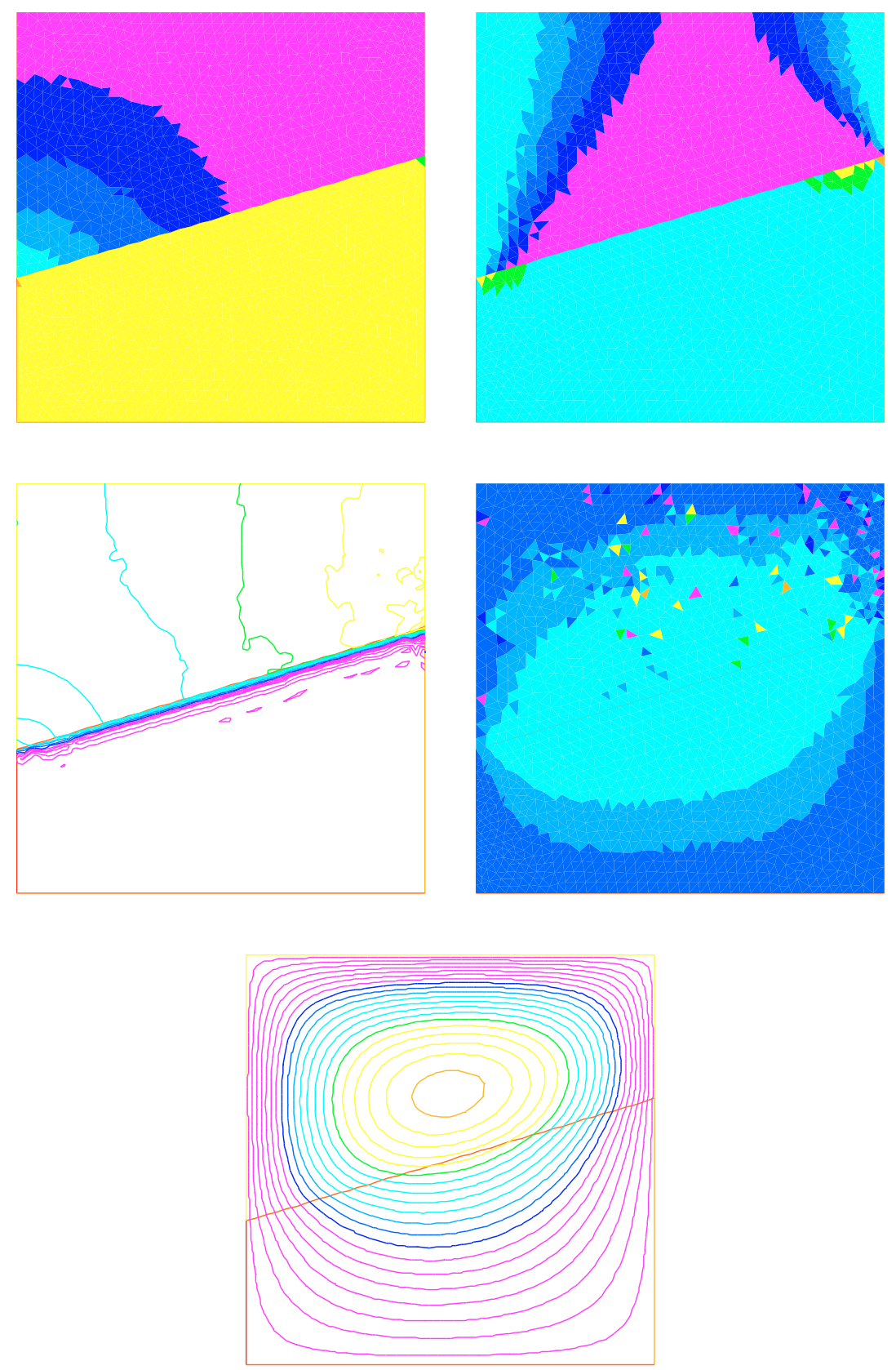

Figure 7. A seeping fluid (porosity 0.01).

Lemma A.1. For all $\varepsilon, 0<\varepsilon<\frac{1}{2}$, there exists a constant $\alpha_{1}(\varepsilon)$ such that, for all e in $\mathcal{E}_{h}^{\Gamma}$,

$$
\left\|\boldsymbol{v}_{h} \cdot \boldsymbol{n}\right\|_{H^{\varepsilon-\frac{1}{2}(e)}} \leq \alpha_{1}(\varepsilon) h_{e}^{-\varepsilon}\left\|\boldsymbol{v}_{h}\right\|_{L^{2}\left(K_{e}^{P}\right)^{d}} .
$$


Proof. We have

$$
\left\|\boldsymbol{v}_{h} \cdot \boldsymbol{n}\right\|_{H^{\varepsilon-\frac{1}{2}(e)}}=\sup _{\varphi \in H^{\frac{1}{2}-\varepsilon}(e)} \frac{\int_{e} \boldsymbol{v}_{h} \cdot \boldsymbol{n} \varphi \mathrm{d} \boldsymbol{\tau}}{\|\varphi\|_{H^{\frac{1}{2}-\varepsilon}(e)}} .
$$

Let $\varphi$ be any function in $H^{\frac{1}{2}-\varepsilon}(e)$. We use an affine transformation $\boldsymbol{F}^{-1}$ that maps $K$ onto the reference triangle $\hat{K}$ and we denote by $\hat{e}$ the image of $e$ by this transformation. Then with obvious notation and by setting $\hat{\boldsymbol{w}}_{h}=\frac{B^{-1 T}}{\left\|B^{-1}\right\|} \hat{\boldsymbol{v}}_{h}$, where $B^{-1 T}$ stands for the transposed matrix of $B^{-1}=D \boldsymbol{F}^{-1}$, we obtain

$$
\int_{e} \boldsymbol{v}_{h} \cdot \boldsymbol{n} \varphi \mathrm{d} \boldsymbol{\tau} \leq c h_{e}^{d-1} \int_{\hat{e}} \hat{\boldsymbol{w}}_{h} \cdot \hat{\boldsymbol{n}} \hat{\varphi} \mathrm{d} \hat{\boldsymbol{\tau}}
$$

The mapping: $\varphi \mapsto \bar{\varphi}$, where $\bar{\varphi}$ denotes the extension by zero of $\varphi$ to $\partial \hat{K}$, is continuous from $H^{\frac{1}{2}-\varepsilon}(\hat{e})$ into $H^{\frac{1}{2}-\varepsilon}(\partial \hat{K})$ with norm $c(\varepsilon)$, and, by the trace theorem, the lifting operator: $\bar{\varphi} \mapsto \bar{\varphi}^{*}$ is continuous from $H^{\frac{1}{2}-\varepsilon}(\partial \hat{K})$ into $H^{1-\varepsilon}(\hat{K})$ and its norm $c^{\prime}$ is bounded independently of $\varepsilon$. So, by using the Bramble-Hilbert inequality, we have

$$
\left|\overline{\hat{\varphi}^{*}}\right|_{H^{1-\varepsilon}(\hat{K})} \leq C(\varepsilon)|\hat{\varphi}|_{H^{\frac{1}{2}-\varepsilon}(\hat{e})} \text {. }
$$

On the other hand, we note that, since $\boldsymbol{v}_{h}$ is divergence-free, $\hat{\boldsymbol{w}}_{h}$ also is in the reference coordinates. This yields

$$
\int_{\hat{e}} \hat{\boldsymbol{w}}_{h} \cdot \hat{\boldsymbol{n}} \hat{\varphi} \mathrm{d} \hat{\boldsymbol{\tau}}=\int_{\partial \hat{K}} \hat{\boldsymbol{w}}_{h} \cdot \hat{\boldsymbol{n}} \overline{\hat{\varphi}} \mathrm{d} \hat{\boldsymbol{\tau}}=\int_{\hat{K}} \hat{\boldsymbol{w}}_{h} \cdot \operatorname{grad} \overline{\hat{\varphi}}^{*} \mathrm{~d} \hat{\boldsymbol{x}}
$$

whence, by using the equivalence of norms on finite-dimensional spaces,

$$
\int_{\hat{e}} \hat{\boldsymbol{w}}_{h} \cdot \hat{\boldsymbol{n}} \hat{\varphi} \mathrm{d} \hat{\boldsymbol{\tau}} \leq\left\|\hat{\boldsymbol{w}}_{h}\right\|_{L^{2}(\hat{K})^{d}}\left|\overline{\hat{\varphi}}^{*}\right|_{H^{1-\varepsilon}(\hat{K})} .
$$

Using (A.5) and going back to $K_{e}^{P}$, we have

$$
\int_{e} \boldsymbol{v}_{h} \cdot \boldsymbol{n} \varphi \mathrm{d} \boldsymbol{\tau} \leq C^{\prime}(\varepsilon) h_{e}^{d-1} h_{K_{e}^{P}}^{-\frac{d}{2}}\left\|\boldsymbol{v}_{h}\right\|_{L^{2}(K)^{d}} h_{e}^{\frac{1}{2}-\varepsilon-\frac{d-1}{2}}\|\varphi\|_{H^{\frac{1}{2}-\varepsilon}(e)} .
$$

Thus the desired result follows from (A.4).

In order to bound the second term in (A.2), we first observe that, on each $e$ in $\mathcal{E}_{h}^{\Gamma}$,

$$
\left\|\mu_{h}\right\|_{H^{\frac{1}{2}-\varepsilon}(e)} \leq\left\|\mu_{h}\right\|_{H^{\frac{1}{2}}(e)} .
$$

So the desired bound follows from the next lemma.

Lemma A.2. There exists a constant $\alpha_{2}$ such that, for all $e$ in $\mathcal{E}_{h}^{\Gamma}$ and all $\mu_{h}$ in $\overline{\mathbb{H}}_{h}\left(\Omega_{F}\right)$,

$$
\left\|\mu_{h}\right\|_{H^{\frac{1}{2}(e)}} \leq \alpha_{2} h_{e}^{-\frac{1}{2}}\left\|\mu_{h}\right\|_{H^{1}\left(K_{e}^{F}\right)} .
$$

Proof. Here we use the intrinsic form of the norm of $H^{\frac{1}{2}}(e)$, so that, for any constant $\mu_{e}$,

$$
\left\|\mu_{h}\right\|_{H^{\frac{1}{2}(e)}}=\left(\left\|\mu_{h}\right\|_{L^{2}(e)}^{2}+\left|\mu_{h}-\mu_{e}\right|_{H^{\frac{1}{2}(e)}}^{2}\right)^{\frac{1}{2}} .
$$


By using the mapping that sends the reference element $\hat{K}$ onto $K_{e}^{F}$ and denoting by $\hat{e}$ the edge or face of $\hat{K}$ associated with $e$ by this mapping, we have with standard notation

$$
\left\|\mu_{h}\right\|_{H^{\frac{1}{2}(e)}} \leq c\left(h_{e}^{d-1}\left\|\hat{\mu}_{h}\right\|_{L^{2}(\hat{e})}^{2}+h_{e}^{d-2}\left|\hat{\mu}_{h}-\mu_{e}\right|_{H^{\frac{1}{2}(\hat{e})}}^{2}\right)^{\frac{1}{2}} .
$$

Using the trace theorem and the Bramble-Hilbert inequality yields

$$
\left\|\mu_{h}\right\|_{H^{\frac{1}{2}(e)}} \leq c\left(h_{e}^{d-1}\left\|\hat{\mu}_{h}\right\|_{H^{1}(\hat{K})}^{2}+h_{e}^{d-2}\left|\hat{\mu}_{h}\right|_{H^{1}(\hat{K})}^{2}\right)^{\frac{1}{2}}
$$

Then going back to $K_{e}^{F}$ gives the desired estimate.

Due to the definition of $h_{\min }^{\Gamma}$, estimate (A.1) is a direct consequence of Lemmas A.1 and A.2, together with (A.2) and (A.7).

Remark A.3. The dependency of $\alpha(\varepsilon)$ with respect to $\varepsilon$ is only linked to the norm of the extension by zero from $H^{\frac{1}{2}-\varepsilon}(\hat{e})$ into $H^{\frac{1}{2}-\varepsilon}(\partial \hat{K})$ and it is checked in ([7], Rem. 2.10), that this constant is smaller than $c \varepsilon^{-1}$. So taking $\varepsilon$ equal to $\left|\log h_{\min }^{\Gamma}\right|^{-1}$ leads to the improved estimate, for a constant $\alpha^{*}$,

$$
\forall \mu_{h} \in \overline{\mathbb{H}}_{h}\left(\Omega_{F}\right), \quad \int_{\Gamma} \boldsymbol{v}_{h} \cdot \boldsymbol{n} \mu_{h} \mathrm{~d} \boldsymbol{\tau} \leq \alpha^{*}\left(h_{\min }^{\Gamma}\right)^{-\frac{1}{2}}\left|\log h_{\min }^{\Gamma}\right|\left\|\boldsymbol{v}_{h}\right\|_{L^{2}\left(\Omega_{P}\right)^{d}}\left\|\mu_{h}\right\|_{H_{h}^{1}\left(\Omega_{F}\right)}
$$

\section{REFERENCES}

[1] Y. Achdou, C. Bernardi and F. Coquel, A priori and a posteriori analysis of finite volume discretizations of Darcy's equations. Numer. Math. 96 (2003) 17-42.

[2] M. Amara, D. Capatina-Papaghiuc, E. Chacón-Vera and D. Trujillo, Vorticity-velocity-pressure formulation for Navier-Stokes equations. Comput. Vis. Sci. 6 (2004) 47-52.

[3] C. Amrouche, C. Bernardi, M. Dauge and V. Girault, Vector potentials in three-dimensional nonsmooth domains. Math. Meth. Appl. Sci. 21 (1998) 823-864.

[4] C. Bègue, C. Conca, F. Murat and O. Pironneau, Les équations de Stokes et de Navier-Stokes avec des conditions aux limites sur la pression. Nonlinear Partial Differ. Equ. Appl., Collège de France Seminar IX (1988) 179-264.

[5] C. Bernardi, C. Canuto and Y. Maday, Un problème variationnel abstrait. Application d'une méthode de collocation pour les équations de Stokes. C.R. Acad. Sci. Paris série I 303 (1986) 971-974.

[6] C. Bernardi, C. Canuto and Y. Maday, Generalized inf-sup condition for Chebyshev spectral approximation of the Stokes problem. SIAM J. Numer. Anal. 25 (1988) 1237-1271.

[7] S. Bertoluzza and V. Perrier, The mortar method in the wavelet context. ESAIM: M2AN 35 (2001) 647-673.

[8] D. Braess and R. Verfürth, A posteriori error estimators for the Raviart-Thomas element. SIAM J. Numer. Anal. 33 (1996) $2431-2444$.

[9] D.-G. Calugaru, Modélisation et simulation numérique du transport de radon dans un milieu poreux fissuré ou fracturé. Problème direct et problèmes inverses comme outils d'aide à la prédiction sismique, Thesis, Université de Franche-Comté, Besançon (2002).

[10] M. Crouzeix and P.-A. Raviart, Conforming and nonconforming finite element methods for solving the stationary Stokes equations. RAIRO Anal. Numér. 7 (1973) 33-76.

[11] M. Discacciati, E. Miglio and A. Quarteroni, Mathematical and numerical models for coupling surface and groundwater flows. Appl. Numer. Math. 43 (2002) 57-74.

[12] M. Discacciati and A. Quarteroni, Analysis of a domain decomposition method for the coupling of Stokes and Darcy equations, in Proc. of ENUMATH, F. Brezzi Ed., Springer-Verlag (to appear).

[13] M. Discacciati and A. Quarteroni, Convergence analysis of a subdomain iterative method for the finite element approximation of the coupling of Stokes and Darcy equations. Comput. Vis. Sci. 6 (2004) 93-104.

[14] F. Dubois, Vorticity-velocity-pressure formulation for the Stokes problem. Math. Meth. Appl. Sci. 25 (2002) 1091-1119.

[15] F. Dubois, M. Salaün and S. Salmon, First vorticity-velocity-pressure scheme for the Stokes problem, Internal Report 356, Institut Aérotechnique, Conservatoire National des Arts et Métiers, France (2002) (submitted). 
[16] P.J. Frey and P.-L. George, Maillages, applications aux éléments finis. Hermès, Paris (1999).

[17] P.-L. George and F. Hecht, Nonisotropic grids. Handbook of Grid Generation, J.F. Thompson, B.K. Soni \& N.P. Weatherhill Eds., CRC Press (1998).

[18] V. Girault and P.-A. Raviart, Finite Element Methods for Navier-Stokes Equations, Theory and Algorithms. Springer-Verlag (1986)

[19] F. Hecht, Construction d'une base de fonctions $P_{1}$ non conforme à divergence nulle dans $\mathbb{R}^{3}$. RAIRO Anal. Numér. $\mathbf{1 5}$ (1981) $119-150$.

[20] F. Hecht and O. Pironneau, FreeFem++, see www.freefem.org.

[21] H. Kawarada, E. Baba and H. Suito, Effects of spilled oil on coastal ecosystems, in the Proceedings of European Congress on Computational Methods in Applied Sciences and Engineering 2000, CD-ROM proceedings (2001).

[22] H. Kawarada, E. Baba and H. Suito, Effects of wave breaking action on flows in tidal-flats, in Computational Fluid Dynamics for the 21st Century, M. Hafez, K. Morinishi and J. Périaux Eds., Springer. Notes on Numerical Fluid Mechanics 78 (2001) $275-289$.

[23] W.J. Layton, F. Schieweck and I. Yotov, Coupling fluid flow with porous media flow, Preprint of the University of Magdebourg, report $\mathrm{N}^{\circ} 22-01(2001)$.

[24] J.-C. Nedelec, Mixed finite elements in $\mathbb{R}^{3}$. Numer. Math. 35 (1980) 315-341.

[25] R.A. Nicolaides, Existence, uniqueness and approximation for generalized saddle point problems. SIAM J. Numer. Anal. 19 (1982) 349-357.

[26] P.-A. Raviart and J.-M. Thomas, A mixed finite element method for second order elliptic problems, Mathematical Aspects of Finite Element Methods. Springer, Berlin. Lect. Notes Math. 606 (1977) 292-315.

[27] S. Salmon, Développement numérique de la formulation tourbillon-vitesse-pression pour le problème de Stokes. Thesis, Université Pierre et Marie Curie, Paris (1999).

[28] R. Temam, Theory and Numerical Analysis of the Navier-Stokes Equations. North-Holland (1977).

[29] R. Verfürth, A Review of A Posteriori Error Estimation and Adaptive Mesh-Refinement Techniques. Wiley \& Teubner (1996).

To access this journal online:

www.edpsciences.org 